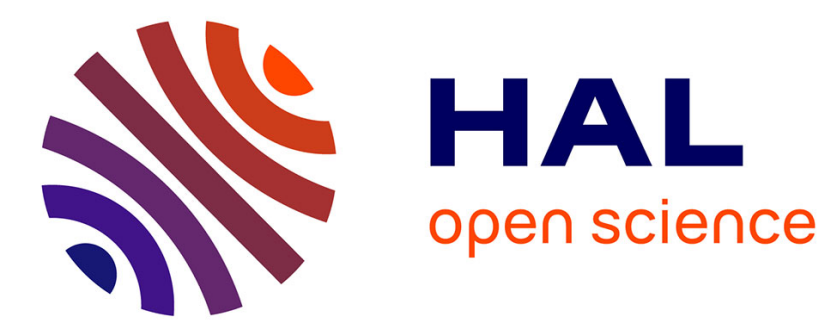

\title{
Vessel Structure Extraction using Constrained Minimal Path Propagation
}

\author{
G. Yang, T. Lv, Y. Shen, S. Li, J. Yang, Y. Chen, H. Shu, L. Luo, J.-L.
}

Coatrieux

\section{- To cite this version:}

G. Yang, T. Lv, Y. Shen, S. Li, J. Yang, et al.. Vessel Structure Extraction using Constrained Minimal Path Propagation. Artificial Intelligence in Medicine, 2020, 105, pp.101846. 10.1016/j.artmed.2020.101846 . hal-02865284

\section{HAL Id: hal-02865284 https://hal-univ-rennes1.archives-ouvertes.fr/hal-02865284}

Submitted on 15 Jun 2020

HAL is a multi-disciplinary open access archive for the deposit and dissemination of scientific research documents, whether they are published or not. The documents may come from teaching and research institutions in France or abroad, or from public or private research centers.
L'archive ouverte pluridisciplinaire HAL, est destinée au dépôt et à la diffusion de documents scientifiques de niveau recherche, publiés ou non, émanant des établissements d'enseignement et de recherche français ou étrangers, des laboratoires publics ou privés. 


\title{
Vessel Structure Extraction using Constrained Minimal Path Propagation
}

\author{
Guanyu Yang ${ }^{1,2}$, Tianling Lv ${ }^{1}$, Yunpeng Shen ${ }^{1}$, Shuo Li ${ }^{3,4}$, Jian Yang ${ }^{5}$, Yang Chen ${ }^{1,2}$, Huazhong Shu ${ }^{1,2}$, Limin \\ Luo $^{1,2}$, Jean-Louis Coatrieux ${ }^{2}$, \\ 1. Laboratory of Image Science and Technology, Southeast University, Nanjing, China; \\ 2. Centre de Recherche en Information Biomedicale Sino-Francais (LIA CRIBs), Rennes, France; \\ 3. Department of Medical Imaging, Western University, London, ON, Canada; \\ 4. Digital Image Group of London, London, ON, Canada; \\ 5. Key Laboratory of Photoelectronic Imaging Technology and System, Ministry of Education, China;
}

Highlights:

- A strategy for a complete vessel extraction is provided under the

framework of minimal path propagation.

- Two constraints (potential constraint

and radius constraint) are devised to

provide efficient vessel extraction.

- The close loop problem in the MPP-BT

algorithm is solved by applying a local

MPP-BT operation.

\begin{abstract}
Minimal path method has been widely recognized as an efficient tool for extracting vascular structures in medical imaging. In a previous paper, a method termed minimal path propagation with backtracking (MPP-BT) was derived to deal with curve-like structures such as vessel centerlines. A robust approach termed
\end{abstract}

CMPP (constrained minimal path propagation) is here proposed to extend this work. The proposed method utilizes another minimal path propagation procedure to extract the complete vessel lumen after the centerlines have been found. Moreover, a process named local MPP-BT is applied to handle structure missing caused by the so-called close loop problems. This approach is fast and unsupervised with only one roughly set start point required in the whole process to get the entire vascular structure. A variety of datasets, including 2D cardiac angiography, 2D retinal images and 3D kidney CT angiography, are used for validation. A quantitative evaluation, together with a comparison to recently reported methods, is performed on retinal images for which a ground truth is available. The proposed method leads to specificity (Sp) and sensitivity (Se) values equal to 0.9750 and 0.6591. This evaluation is also extended to 3D synthetic vascular datasets and shows that the specificity (Sp) and sensitivity (Se) values are higher than 0.99. Parameter setting and computation cost are analyzed in this paper.

Keyword: Minimal path approach, backtracking, vascular structures, segmentation.

\section{INTRODUCTION}

$\mathrm{V}$ ESSEL segmentation is a key step toward accurate visualization, diagnosis and quantification of vascular pathologies. However, vessel segmentation remains a challenging problem because blood vessels often exhibit large structure variability (size/curvature), and their geometrical appearances can be significantly perturbed by stents, stenoses, calcifications, aneurysms and other neighboring anatomical entities, etc. [1][2]. Moreover, challenges in vessel extraction vary from domain to domain. 2-D images like retinal vessel images may face problems like close-loop which most 3-D images do not have. For coronary arteries, we need to distinguish them from atrial ventricles while spines become the problem for aorta segmentation.

Numerous segmentation methods have been proposed to extract vessels of different organ structures and from major imaging modalities [3]-[5]. They can be divided into four categories: filter-based algorithms, model-based algorithms, supervised algorithms and centerline-tracking algorithms. The filter-based algorithms enhance first the vascular structures using a Hessian-matrix-based filter and then segment the vessels on the enhanced image. A typical example is the Frangi filter proposed in [6]. However, although easy to implement, 
such approaches do not consider the vessel topology information and the segmentation accuracy is not ensured. Moreover, all pixels in the image need to be processed by the filter so that the computational time is significantly increased. The model-based algorithms include methods utilizing active contour models [7]-[11]. These algorithms are able to extract vascular structures well, but they are sensitive to the initialization. Image matting model [12] is also applied to retinal vessel segmentation task and achieves outstanding performance. Supervised algorithms [13]-[15] make use of machine learning and can achieve higher segmentation accuracies than other algorithms. Recent deep-learning based algorithms [16]-[18] also show great potential in vessel segmentation tasks. However, hand-crafted labels are needed in those supervised methods which are difficult to acquire. Centerline-tracking algorithms [19]-[32] firstly extract vessel centerlines using techniques like minimal path tracking and then dilate the centerlines to acquire the vessel areas. Their speed and their ability to avoid being caught in local extrema make them very attractive. They are also able to overcome vessel crossing, intensity or width variations as observed in severe stenoses or image degradations [23]. Several manually set points are needed in these algorithms as the start or end points to control the tracking process.

In [30], an algorithm termed minimal path propagation with backtracking (MPP-BT) was proposed for vessel centerline extraction. This MPP-BT algorithm incorporates a backtracking operation into the minimal path propagation to overcome the end-point-setting problem, the shortcut problem and the accumulation problem encountered in standard minimal path techniques. The MPP-BT algorithm realizes the extraction of curve-like structures with only one coarsely set start point and can be effectively used in vessel centerline extraction. However, the MPP-BT algorithm does not offer solution to the extraction of the vessel lumen. In the present work, we aim to propose a method suitable to different imaging modality with features shared by different vessels. An algorithm named constraint-minimal path propagation (CMPP) is developed to fulfill the complete task of vessel extraction within the framework of backtracked minimal path propagation. The performance in centerline extraction is also improved by overcoming the close loop problem via a technique called local MPP-BT operation. Therefore, our contribution includes three elements:

1) A strategy for a complete vessel extraction is provided under the framework of minimal path propagation.

2) Two constraints (potential constraint and radius constraint) are devised to provide efficient vessel extraction.

3) The close loop problem in the MPP-BT algorithm is solved by applying a local MPP-BT operation.

The proposed solution has the following advantages: (i) it is unsupervised, which means that no hand-crafted label is needed in the algorithm; (ii) it is not sensitive to initialization. Only one roughly set start point is needed for initializing the whole process; (iii) it is faster than filter-based algorithms as only pixels inside and around vessel areas are visited.

The rest of this paper is organized as follows. In Section II, we firstly review the backtracking strategy in minimal path propagation and the MPP-BT approach, and then, the proposed CMPP method is introduced in details, including the
'Convexity' and "Symmetric Convexity" metrics (for the vessel and centerline structures). The constrained propagation with automatic stopping, and the solution to the close loop problem are also described. In Section III, the performance of our approach is assessed using 2D X-ray angiography datasets, 2D retinal images and volumetric CT angiography (CTA). A quantitative evaluation on retinal images is provided together with a comparison to other competing methods. An analysis of the computation cost and the parameter sensitivity is also given. In Section IV, some relevant problems are addressed before concluding and sketching future plans.

\section{METHOD}

\subsection{Review of the MPP-BT algorithm}

Minimal path methods extract curve-like structures in image $f$ by searching a path with a contour dependent minimum integrated energy between user-preset start and end points. $E(C)$ is defined as the integrated energy along a path $C$ according to a given potential function $P$ :

$$
E(C)=\int_{\Omega} \tilde{P}(C) d s=\int_{\Omega}(P(C(s))+\omega) d s
$$

where, $\Omega$ denotes the data space. $\omega$ is the regularization term (often a real positive constant) and $C(s) \in R^{n}$ is a parameterized path with the arch length $s$. The cost associated to each point in $C$ can be denoted by the cost function $P(C(s))$ , which is calculated in such way that the preferable feature points have smaller values than non-feature points. In this paper, the "Symmetric Convexity" metric in [31] is used to calculate $P$ for the centerline extraction. The minimal action map $U_{p_{0}}(p)$ is defined as the minimum integrated energy among all the possible paths between the start point $p_{0}$ and a point $p$ :

$$
U_{p_{0}}(p)=\inf _{A\left(p_{0}, p\right)}\{E(C)\}=\inf _{A\left(p_{0}, p\right)}\left\{\int_{\Omega} \tilde{P}(C) d s\right\}
$$

where $A\left(p_{0}, p\right)$ denotes the set of all the paths from $p_{0}$ to $p$. The minimal path between the point $p_{0}$ and the point $p$ can be efficiently obtained using the Dijkstra algorithm [33] or fast marching methods [34]. Here, the Dijkstra method is used to solve (2) in the discrete domain. The Dijkstra algorithm can be applied by first setting all the node costs to infinity and then using an explicit discrete front propagation with direction pointing from current minima to its neighboring nodes. At each step in this process, a priority queue is used to find the next propagation point with the smallest $U$ among all reached points ordered in a minimum heap data structure. The $U$ values of the neighboring nodes of each reached point will be calculated and ordered in the minimum heap data structure. With no specified end point, solution to Eq. (2) is in fact a feature-preferred propagation from the only starting point, called minimal path propagation in this study.

As the connection information is stored, we can easily trace back from each reached point $p$ all the previous points $p^{\prime}$ toward the start point $p_{s}$. This is termed backtracking operation. Obviously, the points associated with lower potential 
$P$ are preferably reached in the propagation and are thus more frequently revisited in the backtracking process. Based on the significantly different numbers of revisits upon different structures, a backtracking operation is proposed in [30] to build the MPP-BT algorithm to overcome the end problem, the shortcut problem and the accumulation problem found in standard minimal path based techniques.

An illustration of this backtracking operation is given in Fig.1, where $p_{1}, p_{2}$ and $p_{3}$ represent the three types of points, namely the non-centerline vessel points, the background points and the centerline points. The potential function $P$ is constructed according to [30]. Then, from each reached grid point the MPP-BT algorithm traces back to the point $p_{b k}^{E}$ with a path of a fixed length $l_{b k}$ and performs accumulation with a value inversely proportional to $P\left(p_{b k}^{E}\right)$ upon each $\hat{I}_{B K}\left(p_{b k}^{E}\right)$ in the feature map $\hat{I}_{B K}$ which is initialized to zero for all points. It results that the centerline points can receive more accumulations than the non-centerline points. From Fig.1 (e),

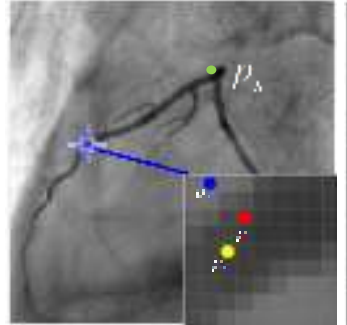

(a)

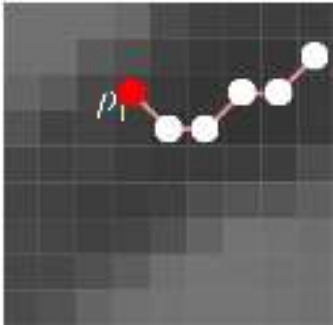

(b)

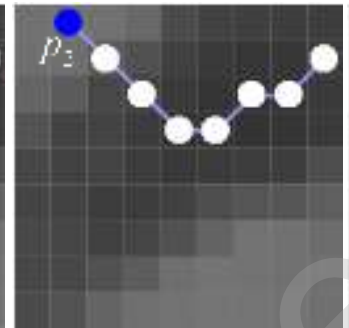

(c) we can see that the points along the vessel centerline will receive much more visits than the points in the backtracking path which are not located on the centerline. To alleviate the accumulation problem caused by the increased cost as the propagation proceeds, the MPP-BT algorithm resets the accumulated cost $U_{p_{0}}(p)$ of each reached point $p$ to the difference between the accumulated costs of the current point $p$ and the last point $p_{b k}^{E}$ in the backtracking path. This operation can make the propagation more effective in identifying more centerline points, especially those far away from the start point. To guarantee a smooth accumulation, we just trace back $l_{b k}$ steps and record the last $l_{b k}$ points [30]. In the MPP-BT algorithm in [30], the minimal path propagation is stopped when a stopping criterion is fulfilled. Considering the fact that most end points in the backtracking paths are also the centerline points with lower cost, the accumulated cost

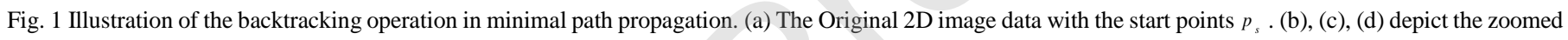

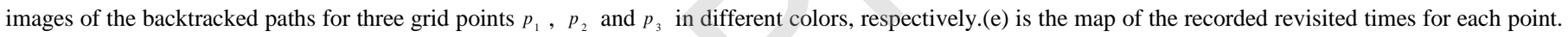
$\left(\kappa=1, \lambda=20, l_{\mathrm{bk}}=15, R_{\min }=2, R_{\max }=15\right.$, according to [30])

difference $\left(U(p)-U\left(p_{b k}^{E}\right)\right)$ along the backtracking path will significantly increase when the propagation starts proceeding into non-vessel regions with much larger $P$ values. Based on this observation, a metric based on the backtracking speed together with a stopping criterion is used in the MPP-BT algorithm to automatically stop the propagation. This will be discussed in section 2.2.2.

\subsection{The proposed CMPP method}

As reported in [30], building the potential $P$ in Eq. (1) is crucial to the performance of the centerline extraction. A metric termed "Symmetric Convexity" was used in MPP-BT algorithm to calculate the potential for the centerline extraction. This "Symmetric Convexity" metric was built based on the "symmetricity" and "convexity" properties of the 1-D intensity distribution across vessel points.

Unlike centerline points, vessel points can be located at any position inside the lumen and do not have such symmetricity constraint. So a medialness metric solely based on the "convexity" property is used for vessel extraction. In this study, we propose an algorithm termed constrained minimal path propagation (CMPP) to get an efficient extraction of the vessel lumen. The "constraint" knowledge in CMPP algorithm is incorporated via the following two forms: the potential constraint and the radius constraint, which can be well built using the information obtained during the centerline extraction step.

\subsubsection{Calculation of 'Convexity' and "Symmetric Convexity" metrics for vessel structures and centerlines}

According to [30], the combination of the "Symmetry" and the "Convexity" properties leads to the "Symmetric Convexity" metric for the centerline points in cost function building. For centerline extraction, the medialness measure $M_{\mathrm{c}}(p, \theta)$ is calculated[31]. Because the points in the lumen do not have the so-called symmetricity property, to perform the vessel extraction, a new medialness measure $M_{v}(p, \theta)$ is used, which only considers the "Convexity" property[31]. Note that $M_{\mathrm{c}}^{s}\left(p, \theta_{n}\right)$ and $M_{\mathrm{v}}^{s}\left(p, \theta_{n}\right)$ are obtained by sorting all the $M_{\mathrm{c}}\left(p, \theta_{i}\right)$ and $M_{\mathrm{v}}\left(p, \theta_{i}\right)$ in descent order with respect to all the orientations, e.g.:

$$
M_{\mathrm{c}}^{s}\left(p, \theta_{n}\right) \geq M_{\mathrm{c}}^{s}\left(p, \theta_{n+1}\right), M_{\mathrm{v}}^{s}\left(p, \theta_{n}\right) \geq M_{\mathrm{v}}^{s}\left(p, \theta_{n+1}\right) .
$$

Fig.2 (b) and (c) illustrate the 2-D measures $M_{\mathrm{c}}(p)$ and $M_{v}(p)$ for one simulated 2-D vessel image in Fig.2 (a). The two measures $M_{\mathrm{c}}(p)$ and $M_{\mathrm{v}}(p)$ are respectively calculated using (3) and (5), with values mapped into [0, 255] for display. We can see in Fig.2 that the two measures $M_{\mathrm{c}}(p)$ 
and $M_{\mathrm{v}}(p)$ give specific highlights of the vessel structures (i.e. centerlines and branches).

\subsubsection{Constrained propagation with automatic stopping}

The fact that the centerline points always lie within the vessel structures means that the potential values of these centerline points can be set to zero as the preset start point. Therefore, the first potential constraint can be easily applied by fixing the energy values of all the extracted centerline points to zero in the entire propagation process. With this potential constraint, all the centerline points can be regarded as start points in the propagation process, and then, the surface will propagate equally into the vessel. This will eliminate the influence of the start point position, leading to a significantly improved propagation efficiency.

The radius constraint is directly related to the radius parameter $R_{\max }$. Here, a centerline distance map is used to record the distance between the point reached and the first centerline point in its backtracking path. All the points in this centerline distance map are initially set to infinity except the centerline points, which are simply set to zero. As the propagation proceeds, the distance value related to each point is updated to the shortest distance between the point and its nearest centerline point. Those points with distances larger than $R_{\max }$ will be excluded from the final vessel extraction result. With this radius constraint, some distant non-vessel points (pixels outside the vascular lumen) with large "convexity" values will not be included into the extracted vessel structures.

With these potential and radius constraints, we can see that the CMPP method affords a vessel-feature preferable traversing, and the vessel structures are segmented by directly marking all traversed points as vessel points. Fig.3 illustrates the extraction results of the simulated vascular image for different traversed point numbers. Fig.3 (b) to Fig.3 (e) are the extraction results when the numbers of traversed points are set to 500, 1000, 2000 and 2706 (which is the traversed point number when stopping criterion is satisfied), respectively. It is also found that the propagation should be stopped when the

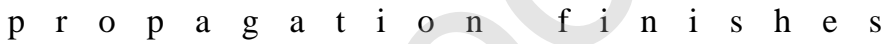
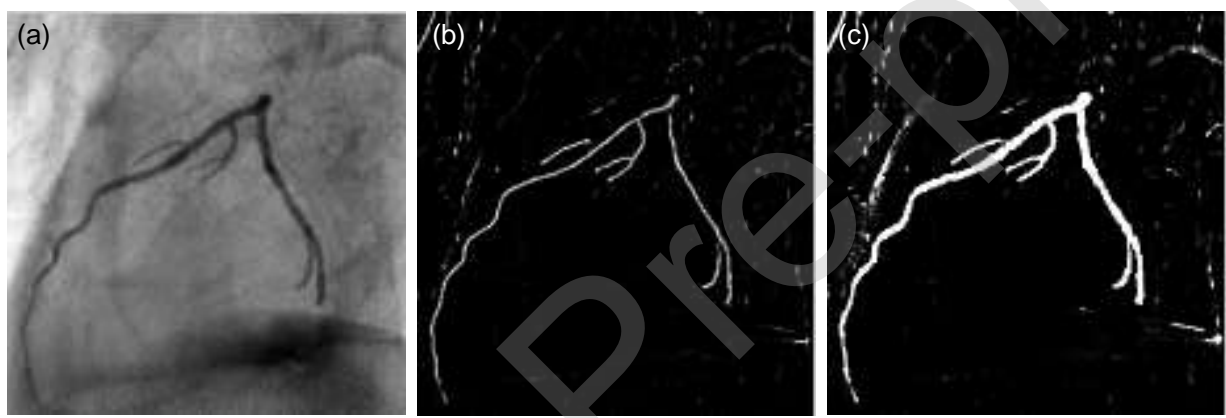

Fig. 2 Illustration of "symmetric convexity"and "convexity" by gray images. (a) Original X-ray simulated image [28]. (b)The"symmetric convexity" gray image. (c)The "convexity" gray image.
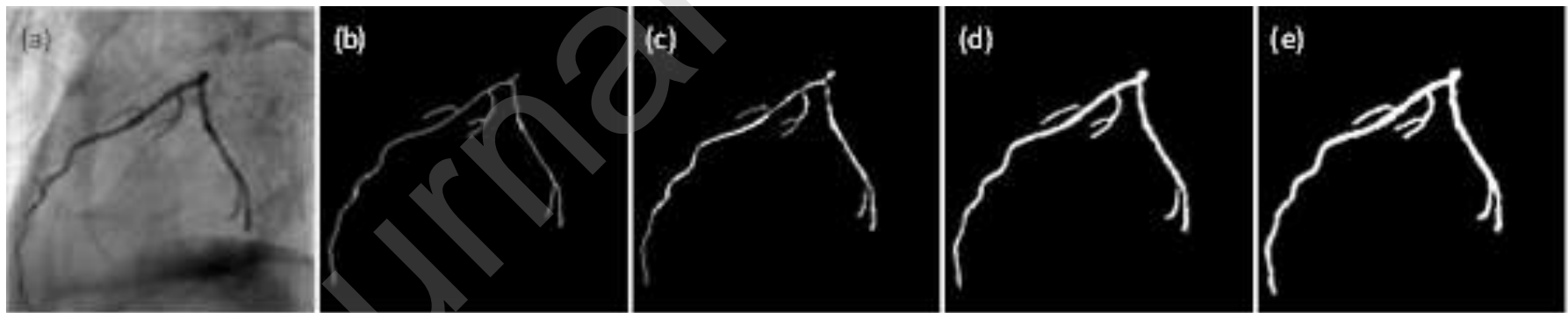

Fig. 3. (a), the original X-ray simulated vascular image. From (b) to (e), the extraction results when the traversed number points in the minimal path propagation are 500,1000,2000, and 2706, respectively. Note that the number 2706 is the recorded traversed point number when the stopping criterion is satisfied. $(\alpha=0.7$, $l_{\mathrm{AVE}}=1000, \kappa=1, \lambda=100, l_{\mathrm{bk}}=15, l_{\mathrm{EC}}=1500, N \bar{S}_{0_{-\mathrm{c}}}^{\text {min }}=0.05, R_{\min }=2, R_{\max }=15$, according to [30])

the traversing of the vessel region. Otherwise, more non-vessel points with large convexity values will be falsely included into the final extracted vessels.

The CMPP method uses two normalized backtracking speed metrics $N \bar{S}_{\mathrm{c}}(p)$ and $N \bar{S}_{\mathrm{v}}(p)$ as the stopping criteria for centerline extraction and vessel extraction, respectively. These two metrics are developed based on the fact that the feature points with lower costs are always preferably visited during backtracking. In consequence, the cost difference along the backtracking path will significantly increase when the propagation starts to pick up non-feature points with much larger energy values after traversing the vascular region. The metrics $N \bar{S}_{\mathrm{c}}(p)$ and $N \bar{S}_{\mathrm{v}}(p)$ are defined as below:

$$
\begin{aligned}
& N \bar{S}_{\mathrm{c}}(p)=\frac{\bar{S}_{\mathrm{c}}\left(p, l_{\mathrm{AVE}}\right)}{S_{\text {max }}(p)} \\
& N \bar{S}_{\mathrm{v}}(p)=\frac{\bar{S}_{\mathrm{v}}\left(p, l_{\mathrm{AVE}}\right)}{S_{\text {max }}(p)}
\end{aligned}
$$




$$
\begin{aligned}
& S_{\mathrm{c}}(p)=\frac{l_{b k}}{U(p)-U\left(p_{b k}^{E}\right)} \\
& S_{\mathrm{v}}(p)=\frac{l_{b k}}{P(p)-P\left(p_{b k}^{E}\right)}
\end{aligned}
$$

where, $p$ is the current point, and $p_{b k}^{E}$ is the point with $l_{b k}$ steps back-traced from the current point $p \cdot \bar{S}_{\mathrm{c}}\left(p, l_{\mathrm{AVE}}\right)$ and $\bar{S}_{\mathrm{v}}\left(p, l_{\mathrm{AVE}}\right)$ in Eq. (7) and Eq. (8) are respectively the average values of $S_{\mathrm{c}}$ and $S_{\mathrm{v}}$ calculated over the $l_{\mathrm{AVE}}$ points reached before the point $p$. One significant difference between Eq. (9) and Eq. (10) is that we use the potential difference rather than the integrated potential difference to calculate $S_{\mathrm{v}}$. With all centerline points' integrated potential set to zero according to the potential constraint, the integrated potential difference is equal to the integrated potential from centerline to $p$, which is unable to distinguish vessel and non-vessel points. That is the reason to use the potential difference here. For the first $l_{\mathrm{AVE}}$ points, $\bar{S}_{\mathrm{c}}\left(p, l_{\mathrm{AVE}}\right)$ and $\bar{S}_{\mathrm{v}}\left(p, l_{\mathrm{AVE}}\right)$ are simply set to the maximum $S_{\mathrm{c}}$ and $S_{\mathrm{v}}$ values. The normalized average backtracking speed metrics $N \bar{S}_{\mathrm{c}}(p)$ and $N \bar{S}_{\mathrm{v}}(p)$ are preferred because the backtracking speed is sensitive to local fluctuations [23]. The normalized backtracking speed $N \bar{S}_{\mathrm{v}}(p)$ for the vessel extraction step runs in the same way as the $N \bar{S}_{\mathrm{c}}(p)$. For the centerline extraction task, the propagation stops when there are $l_{\mathrm{EC}}$ successive points with $N \bar{S}_{\mathrm{c}}$ value lower than a dynamically varying parameter $N \bar{S}_{\mathrm{c}}^{\mathrm{min}} \cdot N \bar{S}_{\mathrm{c}}$ is initially set to an input parameter $N \bar{S}_{0_{-} \mathrm{min}}^{\text {min }}\left(0<N \bar{S}_{0_{-} \mathrm{min}}^{\text {min }}<1\right)$ and is updated to $\min \left\{N \bar{S}_{\mathrm{c}}^{\mathrm{min}}, N \bar{S}_{\mathrm{c}}(p)\right.$ \}every time a new point $p$ is reached. For the vessel extraction, the same process is applied with different parameters. We use respectively $l_{\mathrm{Ev}}, N \bar{S}_{\mathrm{v}}, N \bar{S}_{\mathrm{v}}^{\mathrm{min}}$, $N \bar{S}_{0_{-} v}^{\text {min }}$ instead of $l_{\mathrm{EC}}, N \bar{S}_{\mathrm{c}}, N \bar{S}_{\mathrm{c}}^{\mathrm{min}}, N \bar{S}_{0_{-} \mathrm{m}}^{\mathrm{min}}$.

\subsubsection{Local MPP-BT operation to solve the close loop problem}

During the centerline extraction, the MPP-BT algorithm traces back $l$ steps and performs accumulation upon the last point $p_{b k}^{E}$ in the backtracking path for each reached point. Such accumulation operations on all the last reached points yield the centerline feature map $\hat{I}_{\mathrm{B}}$. Fig.4 (a) and Fig.4 (b) illustrate one retina image (obtained from the retinal database DRIVE [27]), and a zoomed area in blue including one closed loop structure. Fig.4(c) illustrates the traversed area with the direction marked by red arrows in Fig.4(c). Fig.4(d) depicts the expanding propagation of the traversed area at different phases (indicated by dotted red lines). In the MPP-BT algorithm, to be identified as the centerline points, points need to get enough accumulations in the backtracking process. However, and in particular when facing closed loops, some points might get greatly reduced accumulations in the backtracking process because alternative routes exist with less integrated potential. The result is that the points near the blue line (bottom-right in Fig. 4(c)) cannot be accumulated in any point's backtracking path and so, some centerline points near the blue line cannot be extracted from the centerline feature map $\hat{i}_{\text {в }}$. In Fig.4(e), one
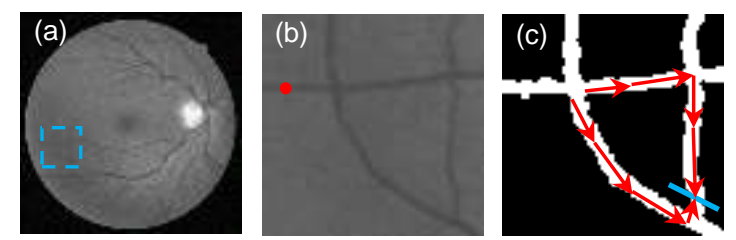

(d)
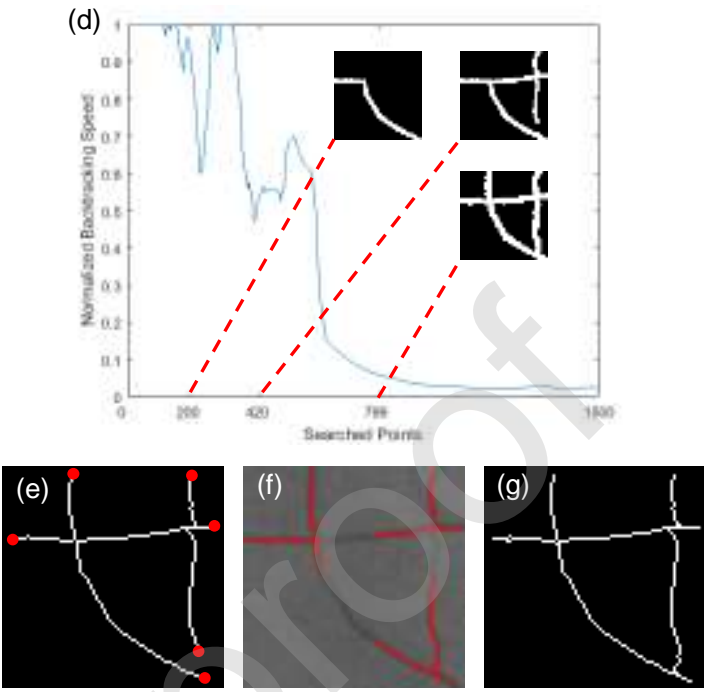

small but obvious discontinuity can be seen when applying the MPP-BT algorithm reported in [30]. We call this problem the close loop problem.

In the present study, to solve this close loop problem, an operation named local MPP-BT operation is performed on the preliminary centerline map $\hat{I}_{\mathrm{C}}$ built from the original MPP-BT algorithm. In the centerline map $\hat{I}_{\mathrm{C}}$, the break points are first identified as those points connected to only one centerline point. These break points in Fig.4 (d) are marked as red points in Fig.4 (e) and they also include the end points of vessel branches. Then, our local MPP-BT operation is applied by implementing the MPP-BT algorithm in [30] using each break point as a start point. When compared to the original MPP-BT algorithm, such local MPP-BT operation appears more efficient because this local MPP-BT operation is stopped after a fixed traversed number $N$. Nevertheless, one local MPP-BT operation is required for each break point, so the combination of all the local MPP-BT operations takes a bit more time than the original MPP-BT method, as we will see in Table 6. In Fig.4 (f), the red lines depict the local centerline results extracted this way and further combined with the centerline image $\hat{I}_{\mathrm{C}}$ in Fig.4 (e) to get the final result illustrated in Fig.4 (g).

For 3-D images like coronary CTA images, the close loop problem is not the main concern as there is no loops. However, local MPP-BT is still valuable in 3-D images. The original MPP-BT is sometimes unable to extract centerline points at vessel endings due to the imprecise stopping criterion. Local MPP-BT can extend the centerlines to make them more complete. 
Fig. 4. Illustration of close loop problem and the solution obtained using the local MPP-BT operation. (a) Original image. (b) The enlarged closed loop structure with a start point marked in red. (c) The propagation area with the arrowed propagation directions for the original MPP-BT algorithm in [30]. (d) Different propagation phases with the corresponding traversed areas. (e) The preliminary extracted centerline map resulting from the original MPP-BT algorithm. (f) The local centerline extraction result overlapped with the original image data. (g) The final extraction obtained by means of the local MPP-BT algorithm. $\left(\alpha=0.7, l_{\mathrm{AVE}}=1000, \kappa=1, \lambda=20, N=2000, l_{\mathrm{EV}}=10000\right.$, $\left.l_{\mathrm{bk}}=10, l_{\mathrm{EC}}=2500, N \bar{S}_{0_{-} \mathrm{c}}^{\min }=0.05, N \bar{S}_{0_{-} \mathrm{v}}^{\text {min }}=0.05, R_{\min }=2, R_{\max }=10\right)$

\subsection{The complete flowchart of the CMPP method}

Fig. 5 summarizes the flowchart of the CMPP method. For each specific vessel, the start point and the parameters being set,the minimal path propagation based method in [30] is run to extract centerlines. The centerline feature map is obtained after the propagation is stopped when the first stopping criterion is met. Then, the local MPP-BT operation is applied to solve close loop problems. After this, with the centerline map, another minimal path propagation using the potential constraint and the radius constraint is carried out to retrieve the complete vessel structures. The finally extracted vessels are obtained by tagging all the traversed point as vessel points when the stopping condition is satisfied.

Fig. 5.The complete flowchart of the CMPP method

Table 1 Parameter setting for the proposed method

\begin{tabular}{|c|c|}
\hline Test data & Parameter Settings \\
\hline $\begin{array}{c}\text { 2-D } \\
\text { coronary } \\
\text { artery }\end{array}$ & $l_{\mathrm{bk}}=15, l_{\mathrm{EC}}=2500, N \bar{S}_{0_{-} \mathrm{c}}^{\text {in }}=0.05, N \bar{S}_{0_{-} \mathrm{v}}^{\min }=0.05, R_{\min }=2, R_{\max }=15$ \\
\hline $\begin{array}{l}2-\mathrm{D} \\
\text { retinal vessel }\end{array}$ & $l_{\mathrm{bk}}=10, l_{\mathrm{EC}}=2500, N{\overline{S_{0_{-} \mathrm{c}}}}_{\mathrm{min}}=0.05, N \bar{S}_{0_{-v}}^{\min }=0.05, R_{\mathrm{min}}=2, R_{\max }=10$ \\
\hline $\begin{array}{l}3-\mathrm{D} \\
\text { coronary } \\
\text { artery }\end{array}$ & $l_{\mathrm{bk}}=15, l_{\mathrm{EC}}=5000, N \bar{S}_{0_{-\mathrm{c}}}^{\text {min }}=0.05, N{\overline{S_{0_{-} \mathrm{v}}}}_{\text {min }}=0.05, R_{\mathrm{min}}=2, R_{\mathrm{max}}=20$ \\
\hline $\begin{array}{c}3-\mathrm{D} \\
\text { kidney artery }\end{array}$ & $l_{\mathrm{bk}}=15, l_{\mathrm{EC}}=5000, N \bar{S}_{0_{-} \mathrm{c}}^{\min }=0.05, N \bar{S}_{0_{-} \mathrm{m}}^{\min }=0.05, R_{\mathrm{min}}=2, R_{\max }=15$ \\
\hline
\end{tabular}

\section{EXPERIMENTS}

\subsection{Data description and parameter setting}

The simulated angiography image (Fig.1 (a)) with intensities normalized into the gray-level range $[0,255]$ is used. This simulation is performed based on [37] by projecting a manually segmented 3-D vessel tree $(256 \times 256 \times 256)$ onto a $256 \times 256$ 2 -D background image. In addition to the simulated image in Fig.1 (a), four real $512 \times 512$ coronary artery angiogram images were collected from a GE rotational angiography system in the Cardiology Department of the University Hospital of Rennes, France. More details on the rotational angiography system can be found in [37]. The 3-D coronary artery datasets (in Fig.9 (a) and (b)) correspond to nine sets of CT angiography (CCTA) about 300 2-D slices. The retinal vessel images used in this paper are obtained from the DRIVE (Digital Retinal Images for Vessel Extraction) database, and another publicy available retinal database STARE (STructured Analysis of the Retina) was used for more detailed evalution of our method. In DRIVE dataset, a total of 40 color fundus photographs together with their manual delineations was used (refer for more details to [35]). STARE consists of 20 images, 10 of them contain pathology [36]. The 3-D kidney artery dataset (see Fig.9(c)) is composed of CT angiography (CCTA) data acquired from a 64 dual-source CT in the Jiangsu Province Hospital, China.

The implementation has been performed on a PC (Intel Core $^{\mathrm{TM}} 4$ Quad CPU and 20 GB RAM, GPU (NVIDIA GTX560)) with Visual C++ as the developing language (Visual Studio 2010 software; Microsoft). The pixel-wise calculations

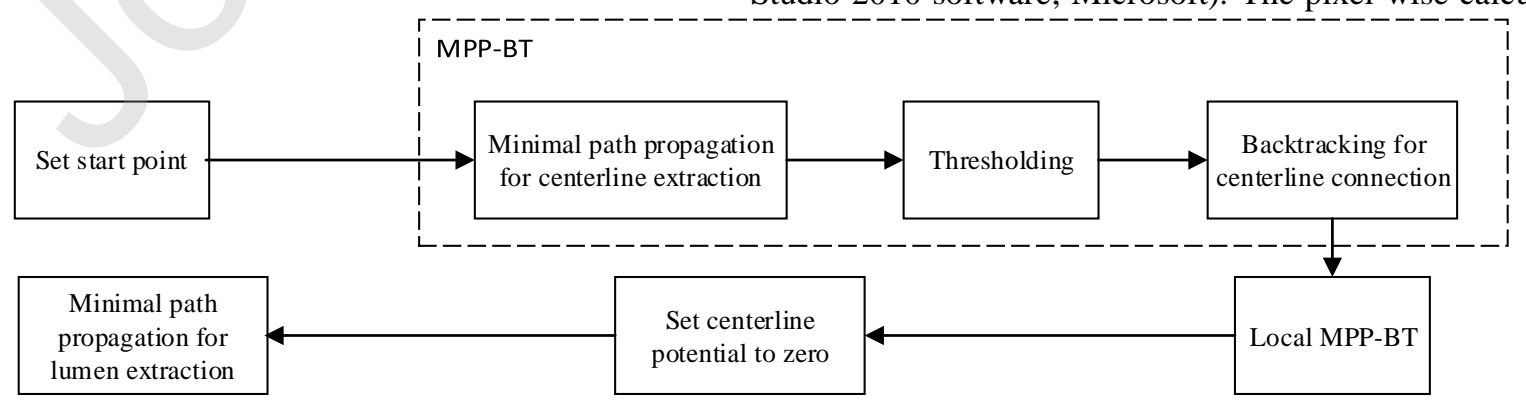

data acquired from a Siemens dual-source CT system (Somatom Definition Flash) in the Radiology Department of the First Hospital of Nanjing, China. Each 3-D CCTA includes of 2-D image data involved in the computation of $M_{\mathrm{c}}(p, \theta)$ and $M_{v}(p, \theta)$ were parallelized using a CUDA-based GPU 
(respectively Compute Unified Device Architecture and
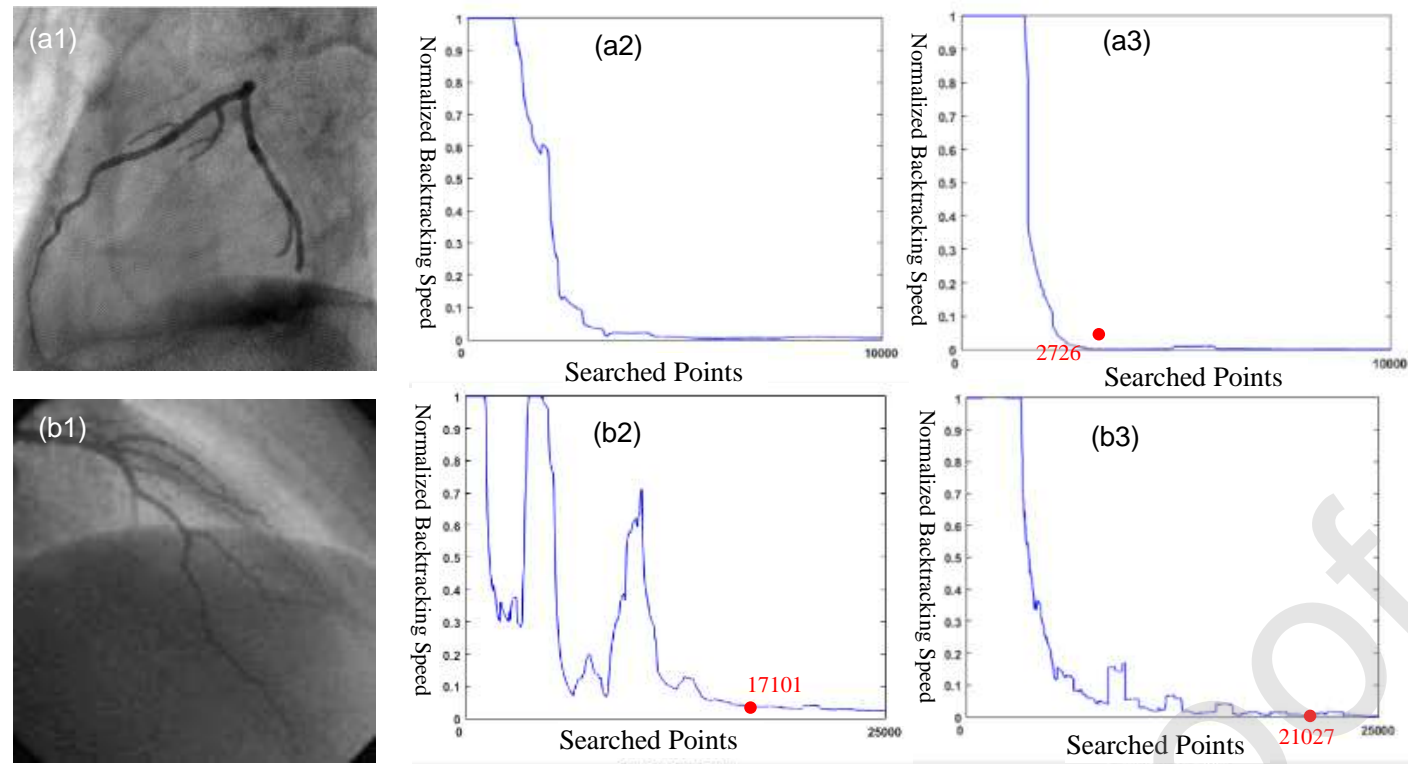

Graphic Processing Unit) [39][40].

Thirteen parameters require to be set in the proposed method. They include $D$ describing the number of directions used for calculating the potential, $l_{\mathrm{bk}}$ limiting the backtracking steps, the coefficient $\alpha$ of quantile value applied in the feature map $\hat{I}_{\mathrm{B}}$ in order to get the intermediate feature image $\hat{I}_{\mathrm{C}}$, the number of propagation steps $N$ modulating the local MPP-BT operations. The propagation stopping mechanism includes the parameter $l_{\mathrm{AVE}}$ for the computation of the normalized average speed, the successive point numbers $l_{\mathrm{EC}}$ (for centerline) and $l_{\mathrm{EV}}$ (for vessel lumen) and the initial values $N \bar{S}_{0_{-} \text {min }}^{\text {min }}$ (for centerline) and $N \bar{S}_{0_{-} v}^{\text {min }}$ (for vessel lumen). The parameters of the "symmetric convexity" metric include $\kappa, \lambda$ and the vessel radius range $\left[R_{\min }, R_{\text {max }}\right]$. Most parameters were selected under the guide of one experienced radiologist (Y.X.D 20 years experience) to provide good extraction results. It is found that all the involved parameter can be robustly set in the proposed CMPP method. The parameters $\alpha, N, l_{\mathrm{AVE}}, \kappa, \lambda$ and $l_{\mathrm{EV}}$ were kept unchanged

Fig. 6. Illustration of the stopping criterion for centerline and vessel structure extraction on two images. Fig.6(a1) and Fig.6 (b1) are the original images; (a2) and (b2) plot the calculated normalized average speed of centerline extraction; (a3) and (b3) plot the calculated normalized average speed of vessel extraction. The number of reached points is marked in red. $\left(\alpha=0.7, l_{\mathrm{AVE}}=1000, \kappa=1, \lambda=20, N=2000, l_{\mathrm{EV}}=10000, l_{\mathrm{bk}}=15, l_{\mathrm{EC}}=2500, N \bar{S}_{0_{-} \mathrm{m}}^{\text {min }}=0.05, N \bar{S}_{0_{-} \mathrm{m}}^{\text {min }}=0.05\right.$,

$\left.R_{\min }=2, R_{\max }=15\right)$

\subsection{Effect of local MPP-BT operation}

Fig.7, Fig.8 and Fig.9 illustrate the extraction results of 2-D coronary artery images, 2-D retinal images and 3-D vascular image datasets (including the 3-D coronary artery datasets and the kidney artery), respectively. The left to right columns in Fig.7-Fig.8 display the original images, the extracted vascular centerline images using the original MPP-BT method, the extracted vascular centerline images using the MPP-BT method with local MPP-BT operations, the extracted vascular structure using the CMPP method, and the manually delineated vascular structure images. The start points are marked in red in the original images. We can see in the second columns in Fig.7, Fig.8 and Fig.9 that the original MPP-BT algorithm fails to extract the full centerline structure due to the close loop for all datasets $\left(\alpha=0.7, l_{\mathrm{AVE}}=1000, \kappa=1, \lambda=20, N=2000\right.$ , $\left.l_{\mathrm{EV}}=10000\right)$. We set $D=2$ for 2-D images while $D=6$ for 3 $\mathrm{D}$ images and the remaining parameters (listed in Table 1) were fixed when processing the datasets of the same type. All these parameters are tuned on one image of each type and validated on other images for evaluation. A detailed analysis of the parameter setting is given and discussed in section 3.6.

\subsection{Effect of the stopping criterion}

The plots in Fig. 6 characterize the behavior of the normalized average backtracking speed $N \bar{S}_{\mathrm{c}}(p)$ (Fig. 6(a2) and (b2)) for centerline propagation stopping and the $N \bar{S}_{v}(p)$ (Fig.6 (a3) and (b3)) for vessel propagation stopping. The stop points (marked as red dots along the $\mathrm{x}$ axis) are displayed in the plots in the middle and right most columns in Fig.6. We can see that the propagation should be stopped to give a good extraction of vessel structure when the normalized average speed $N \bar{S}_{\mathrm{v}}$ is turned to a small value. problem. Much more break points appear in the retinal images which contains many close loop structures.

The arrows in the third column in Fig.7 and Fig.8 show that this close loop problem can be effectively alleviated by applying the local MPP-BT operations. It is found in Fig.7 and Fig.8 that the proposed approach works well in retrieving the complete vascular structures. Nevertheless, in the third columns of Fig.8, we can note that some structures still fail to be extracted even with the local MPP-BT operations (marked as yellow arrows) due to the indistinct intensity feature in the original images.

In Fig.9, the extracted vascular lumens of three kinds of 3-D data are displayed. The columns from left to right represent the original 3-D image data, the extracted centerlines, the extracted 
vessel lumens and the extracted lumens overlaid on the original volumes, respectively. Only the vessel radius range [ $R_{\text {min }}, R_{\max }$ ] needed to be modified while the other parameters were kept unchanged for these different volumes. The first and second rows are illustrations of the left coronary trees and the right coronary trees. The third row displays one kidney artery tree and the last row one carotid artery tree. Results in Fig.9 show that, the proposed CMPP algorithm works well in providing complete 3-D vessel lumen extraction for different kinds of 3D image data.

\subsection{Start point position setting}

To analyze the robustness of the algorithm with respect to the start point position, we compared the extraction results obtained on one vascular image with different initial points. Fig. 10 shows the segmentation results of the proposed CMPP algorithm on 2-D coronary artery images. The rows in Fig. 10 display the original images, the extracted vascular centerlines and vessel structures from top to bottom, respectively. The start points are marked in red in the original images (refer to the left column). It can be seen that the results remain almost the same when the start point is set to different positions inside the vessel lumen or even slightly outside the lumen. The results show that the start point setting is quite robust in the proposed CMPP algorithm.

\subsection{Quantitative Evaluation on $2 D$ vessels}

The DRIVE and STARE database provides a ground truth o f vessel extraction (http://www.isi.uu.nl/Research/Databases/D RIVE/)(http://cecas.clemson.edu/ ahoover/stare/), and a quant itative evaluation can be performed on these public datasets to evaluate the performance of the proposed method for retinal ve ssel images. Let us define $T P$ (true positive) as the right extract ed feature points, $F P$ (false positive) the wrong extracted featu re points, $T N$ (true negative), the correct extracted non-feature (background) points, $F N$ (false negative) as the wrong extracte $\mathrm{d}$ non-feature (background) points.
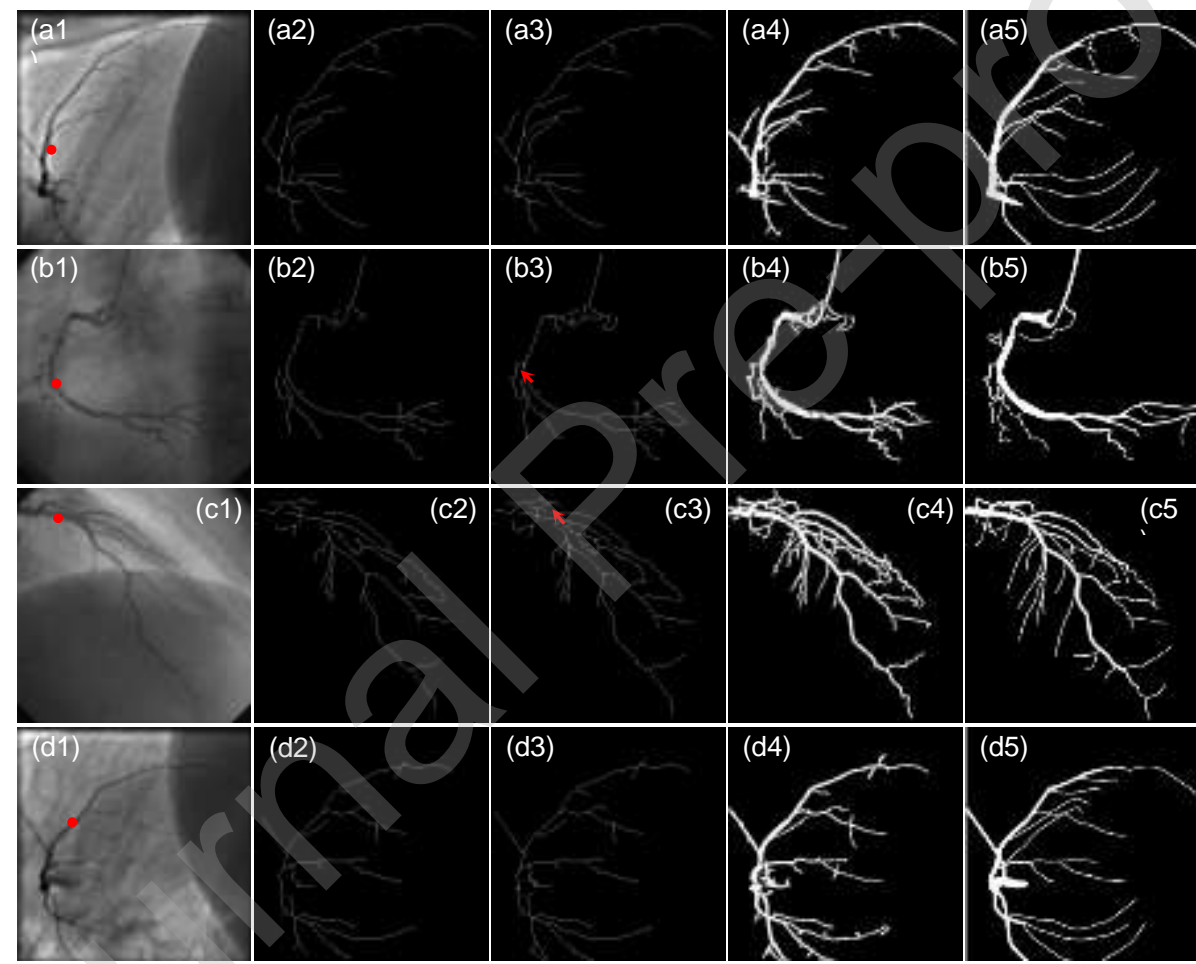

Fig. 7. Extraction result corresponding to four 2-D coronary artery images (from the first row to the fourth row) using the proposed method. From left to right, the first to fifth columns correspond respectively to the original images, the extracted centerlines using the original MPP-BT method in[30], the extracted centerlines using the improved MPP-BT method with local MPP-BT operations, the CMPP extracted vessel structures (in white color), and the manually delineated vessel structures. The starting points are marked by red points in the images in the first column. $\left(\alpha=0.7, l_{\mathrm{AVE}}=1000, \kappa=1, \lambda=20, N=2000\right.$, $\left.l_{\mathrm{EV}}=10000, l_{\mathrm{bk}}=15, l_{\mathrm{EC}}=2500, N \bar{S}_{0 \_\mathrm{c}}^{\min }=0.05, N \bar{S}_{0 \mathrm{v}}^{\min }=0.05, R_{\min }=2, R_{\max }=15\right)$ 

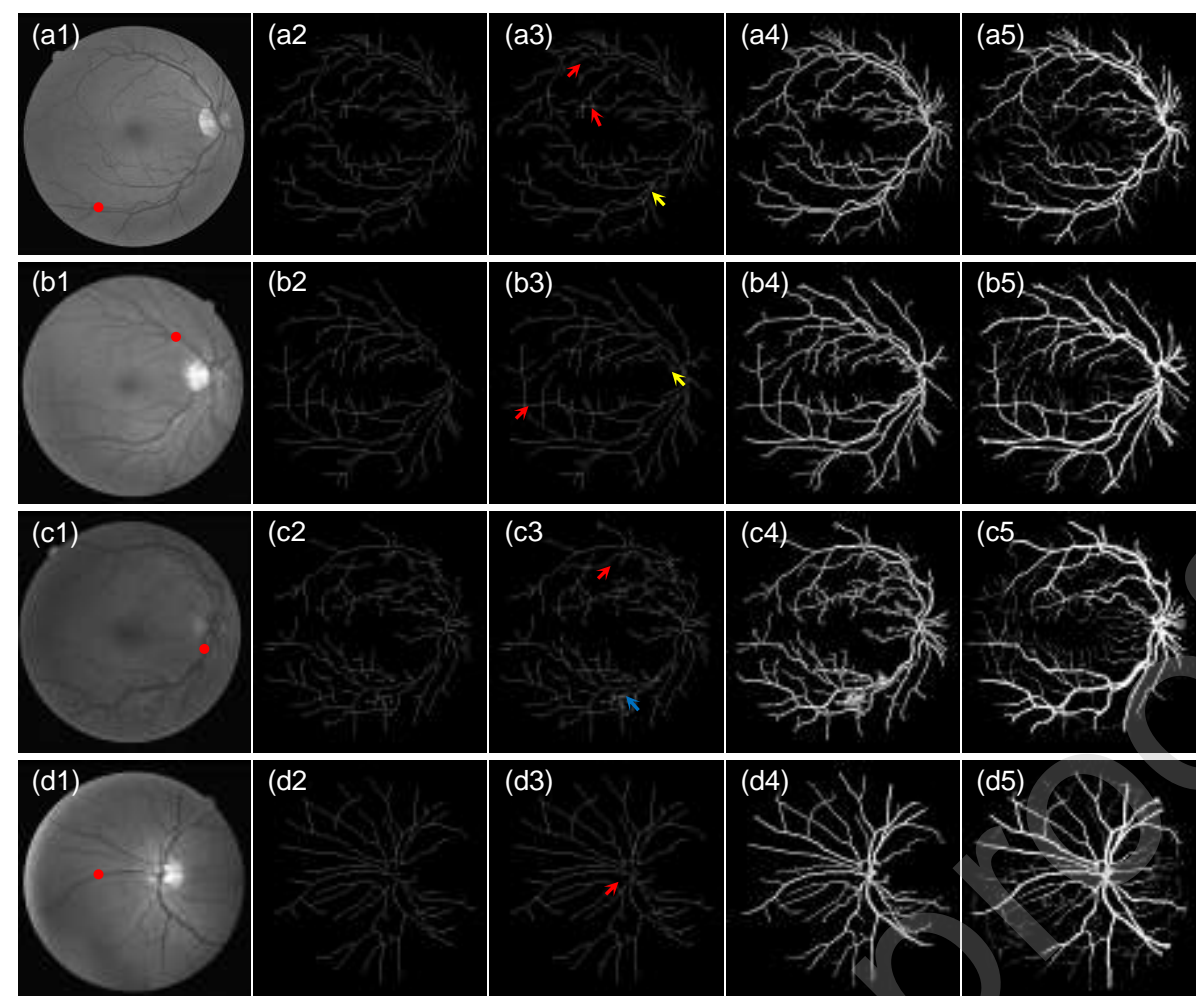

(d3)
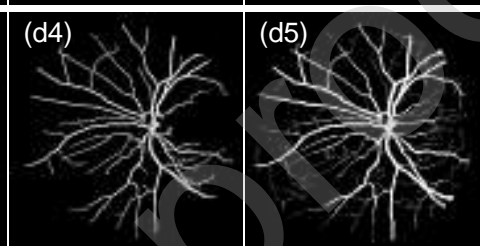

Fig. 8. Extraction result obtained for four retinal images (from the first row to the fourth row) using the proposed method. From left to right, the first, second, third, fourth and fifth columns correspond to the original images, the extracted centerlines using the original MPP-BT method in[30],the extracted centerlines using the improved MPP-BT methodwith local MPP-BT operations, the CMPP extracted vessel structures (in white color), and the manually delineated vessel structures. The starting points are tagged as red points in the images in the first column. $\left(\alpha=0.7, l_{\mathrm{AVE}}=1000, \kappa=1, \lambda=20, N=2000, l_{\mathrm{EV}}=10000, l_{\mathrm{bk}}=10, l_{\mathrm{EC}}=2500\right.$,

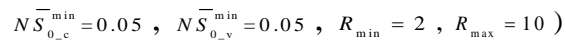
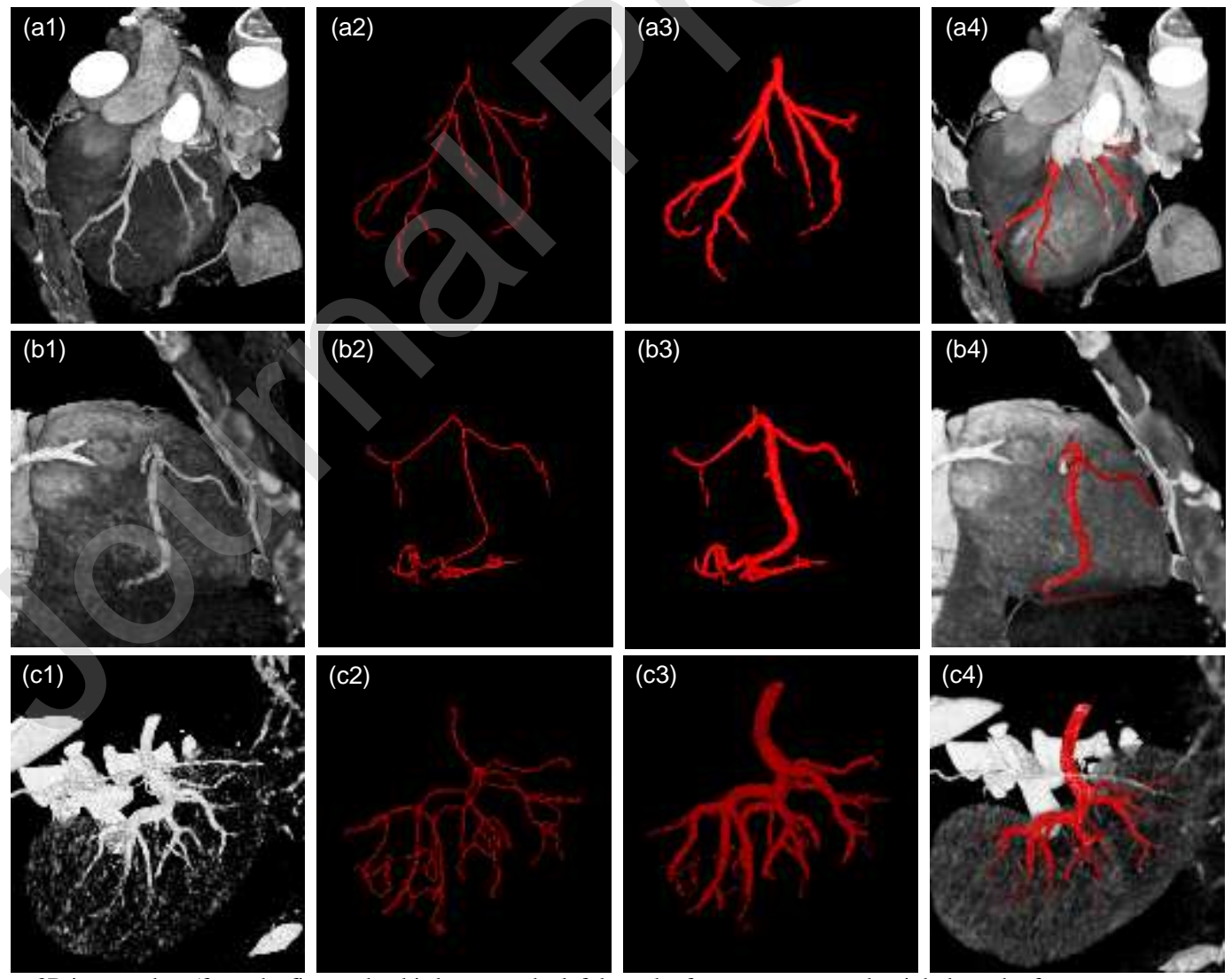

Fig. 9. Application to 3D images data (from the first to the third row are the left branch of coronary artery, the right branch of coronary artery and the kidney artery) using the proposed method. From left to right, the first, second, third and fourth columns correspond to the original images, the extracted centerlines using the improved MPP-BT method with local MPP-BT operations, the extracted vessel structure using the proposed algorithm, and the overlapped vessel images.(For the 


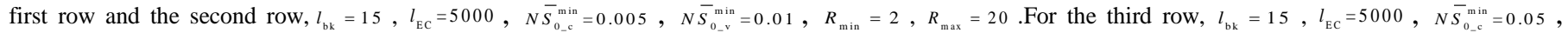
$N \bar{S}_{0_{-v} \text { min }}^{\text {m }}=0.2, R_{\text {min }}=2, R_{\text {max }}=15$ )

Then, the Acc (accuracy), the Se(sensitivity), and the $\mathrm{Sp}$ (specificity) can be expressed by:

$$
\begin{gathered}
\mathrm{Acc}=\frac{T P+T N}{T P+F P+T N+F N}, \\
\mathrm{Se}=\frac{T P}{T P+F N}, \mathrm{Sp}=\frac{T N}{T N+F P}
\end{gathered}
$$

The values of Acc, Se and Sp calculated via Eq.(14) for the extraction results in Fig. 7 and Fig. 8 are listed in Table 2 and Table 3, respectively. The maximum Acc, minimum Acc and average values obtained over the full 40 images of DRIVE database are also listed in the table. In Table 4, a comparison with recent retinal vessel extraction methods is presented.

Table 2. Quantitative Evaluation of the proposed CMPP method on Coronary Images

\begin{tabular}{|c|c|c|c|}
\hline Image(or method) & Acc & Se & Sp \\
\hline Fig.7(a1) & 0.9544 & 0.5536 & 0.9826 \\
\hline Fig.7(b1) & 0.9608 & 0.6933 & 0.9772 \\
\hline Fig.7(c1) & 0.9480 & 0.5714 & 0.9776 \\
\hline Fig.7(d1) & 0.9391 & 0.5621 & 0.9712 \\
\hline Standard deviation & 0.0093 & 0.0659 & 0.0047 \\
\hline
\end{tabular}

We can see that for the segmentation task the proposed method leads to accuracy (Acc) and specificity (Sp) values equal to 0.9345 and 0.9750 , very close to those obtained by the other methods. However, the sensitivity (Se) of the proposed method is found lower than most other methods. This is due to

the vessel point missing caused by the lower "convexity" metric values near the vessel boundaries.

Table 3. Quantitative Evaluation of the proposed CMPP method on Retinal Images

\begin{tabular}{|c|c|c|c|}
\hline Image(or method) & Acc & Se & Sp \\
\hline Fig.8(a1) & 0.9438 & 0.6979 & 0.9809 \\
\hline Fig.8(b1) & 0.9557 & 0.6722 & 0.9881 \\
\hline Fig.8(c1) & 0.9144 & 0.6370 & 0.9618 \\
\hline Fig.8(d1) & 0.9360 & 0.6023 & 0.9873 \\
\hline Maximum Acc & 0.9553 & 0.7652 & 0.9813 \\
\hline Minimum Acc & 0.9144 & 0.6370 & 0.9618 \\
\hline average & 0.9345 & 0.6591 & 0.9750 \\
\hline Standard deviation & 0.0125 & 0.0397 & 0.0115 \\
\hline
\end{tabular}

Table 4. Quantitative Evaluation Results of different retinal vessel extraction methods on DRIVE and STARE database

\begin{tabular}{|c|c|c|c|c|}
\hline Dataset & Method & Acc & Se & Sp \\
\hline \multirow{5}{*}{ DRIVE } & Staal $^{[13]}$ & 0.9441 & 0.7193 & 0.9773 \\
\cline { 2 - 5 } & Marín $^{[14]}$ & 0.9452 & 0.7067 & 0.9801 \\
\cline { 2 - 5 } & Chaudhuri $^{[41]}$ & 0.8894 & 0.2716 & 0.9794 \\
\cline { 2 - 5 } & Zhang $^{[42]}$ & 0.9382 & 0.7120 & 0.9724 \\
\cline { 2 - 5 } & Espona $^{[43]}$ & 0.9352 & 0.7436 & 0.9615 \\
\cline { 2 - 5 } & Mendonca $^{[44]}$ & 0.9452 & 0.7344 & 0.9764 \\
\cline { 2 - 5 } & MSLTA $^{[45]}$ & 0.9285 & 0.7468 & 0.9551 \\
\cline { 2 - 5 } & Proposed $_{5}$ & 0.9345 & 0.6591 & 0.9750 \\
\hline & Marín $^{[14]}$ & 0.9526 & 0.6944 & 0.9819 \\
\cline { 2 - 5 } STARE & Zhang $^{[42]}$ & 0.9474 & 0.7346 & 0.9726 \\
\cline { 2 - 5 } & Mendonca & \\
\cline { 2 - 5 } & Proposed & 0.9440 & 0.6996 & 0.9734 \\
\cline { 2 - 5 } & Propos $^{[44]}$ & 0.9589 & 0.6190 & 0.9911 \\
\hline
\end{tabular}
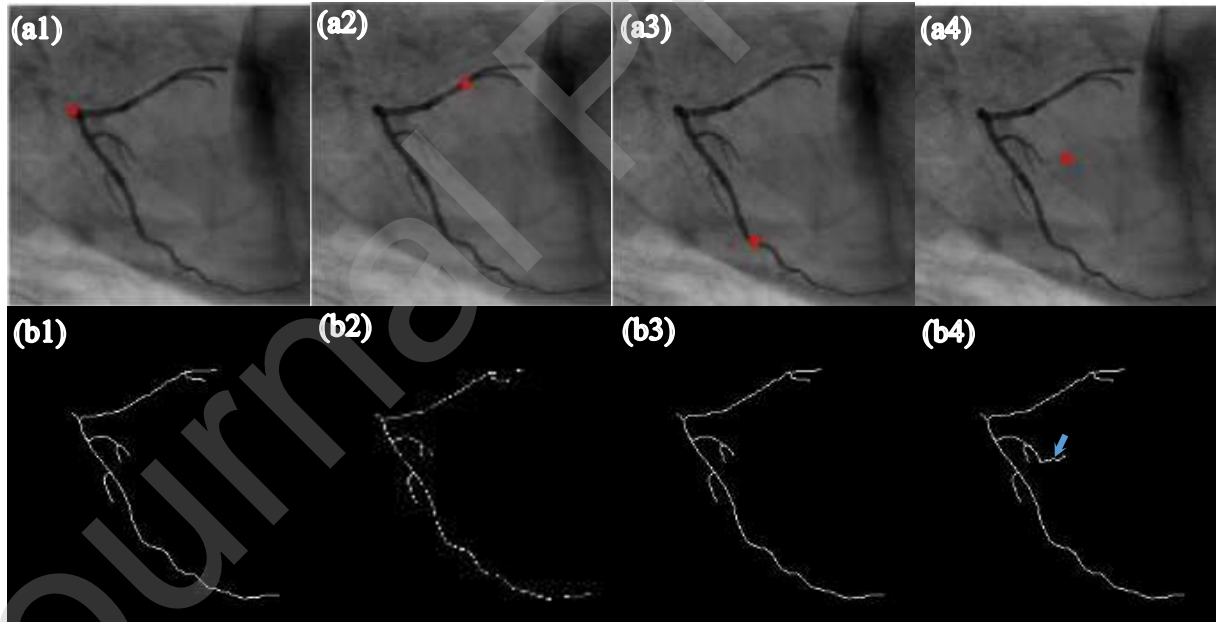

(b2)

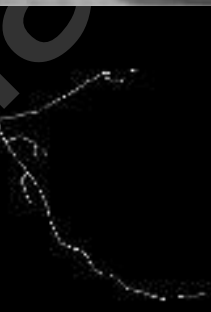

(b3)

(b4)

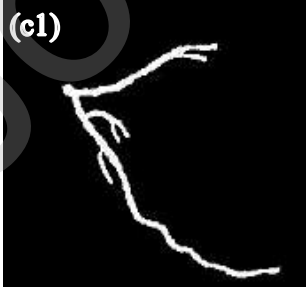

(c2)

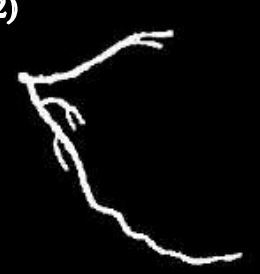

(c3)
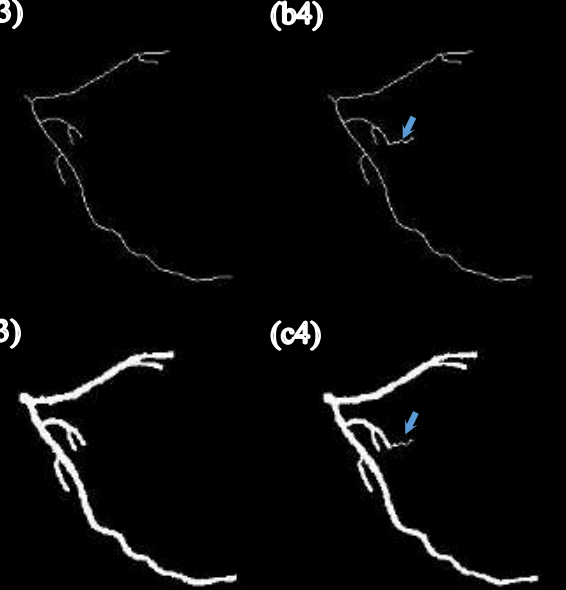

(c4)

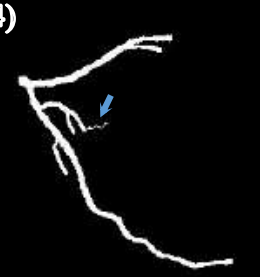

Fig. 10. Results on the same image with different start point setting. Images in the first row are the original images. Start point was set at the vascular root in the first column while at other position inside the vessel in the second and the third column. Start point was set outside the vessel lumen in the fourth column. The red points represents the start point setting. Second row represents the centerline extraction results. Third row represents the vessel segmentation results. Blue arrows show some errors result from setting start point outside the vessel lumen. 


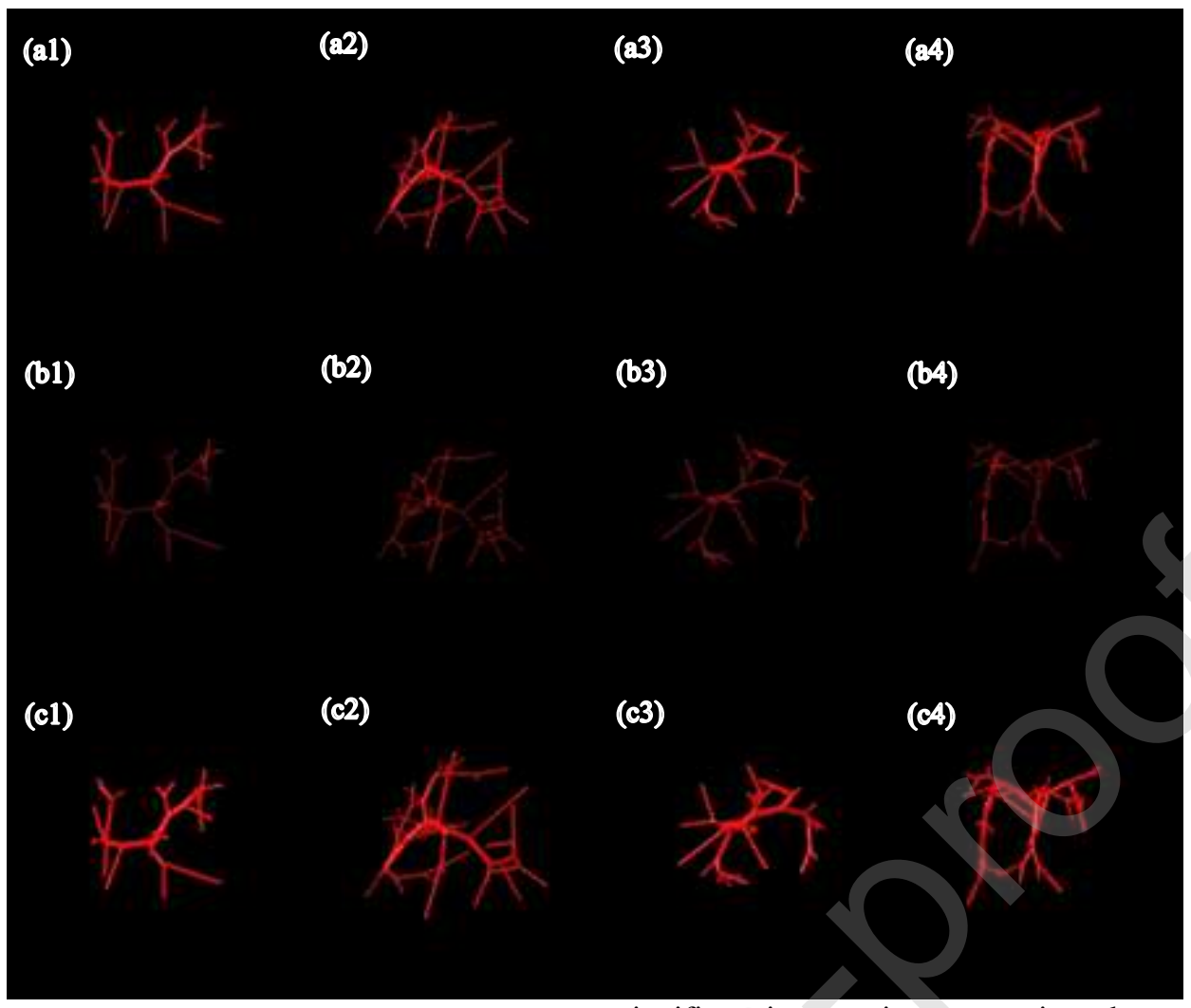

\subsection{Quantitative Evaluation on 3D synthetic images}

In this experiment, we performed a quantitative evaluation of our CMPP Algorithm using synthetic images from VascuSynth dataset (http://vascusynth.cs.sfu.ca/Data.html) which were generated by the method proposed in [46]. The algorithm evaluation criteria are still Acc(accuracy), Se(sensitivity), and Sp (specificity) defined in section 3.5 above and are calculated via Eq.(14). The results are shown in Fig. 11 and Table 5. It can be seen that all Acc and Se values are higher than 0.99 with extremely low standard deviations while the values of $\mathrm{Sp}$ reach 1 for all tested images.

Table 5. Quantitative Evaluation of the proposed CMPP method on Synthetic 3D Data

\begin{tabular}{|c|c|c|c|}
\hline Synthetic 3D Data \\
\hline Image(or method) & Acc & Se & Sp \\
\hline Fig.11(a1) & 0.999972 & 0.9928 & 1 \\
\hline Fig.11(b1) & 0.999907 & 0.9978 & 1 \\
\hline Fig.11(c1) & 0.999980 & 0.9938 & 1 \\
\hline Fig.11(d1) & 0.999993 & 0.9985 & 1 \\
\hline Standard deviation & $3.8323 \mathrm{e}-05$ & 0.0028 & 0 \\
\hline
\end{tabular}

\subsection{Computation Cost}

The vessel structures only occupy a small portion of the total image space and so the CMPP method leads to higher efficiency when compared to filter based methods requiring calculations over all grid points. Table 6 provides a comparison of the average time consumption for the coronary and retinal images displayed in Fig.7 and Fig.8 using Frangi filter [6] and the proposed CMPP algorithm respectively. The computation time of CMPP is smaller which means that the former algorithm is more efficient for the vessel structures extraction. The computation times to process the images displayed in Fig.7, Fig. 8 and Fig.9 are reported in Table 7 and Table 8. The local MPP-BT operation is not used for 3D data in order to avoid the significant increase in computations due to the large number of break points. The vessel structure extraction is quite efficient since all the traversed grid points are directly included into the extracted vessel structures. For one coronary artery image with $512 \times 512$ pixels, the vessel extraction takes less than $0.5 \mathrm{~s}$ while for the retinal case, the time required is less than $1.5 \mathrm{~s}$.

Table 6. Comparison of computation time for 2-D image data using Frangi and CMPP algorithms

\begin{tabular}{|c|c|c|}
\hline \multirow{2}{*}{$\begin{array}{c}\text { D IMAGE } \\
\text { DATA }\end{array}$} & \multicolumn{2}{|c|}{ Average Time(s) } \\
\cline { 2 - 3 } Coronary & 0.8637 & CMPP \\
\hline Retinal & 1.59 & 0.3025 \\
\hline
\end{tabular}

Table 7. Computation time (in seconds) for 2-D image data

\begin{tabular}{|c|c|c|c|c|c|c|c|}
\hline \multirow{2}{*}{\begin{tabular}{c} 
2D \\
IMAGE \\
\cline { 2 - 8 } DATA
\end{tabular}} & \multicolumn{3}{|c|}{ Original MPPBT } & \multicolumn{2}{c|}{$\begin{array}{c}\text { Local } \\
\text { MPPBT }\end{array}$} & \multicolumn{2}{c|}{ CMPP } \\
\cline { 2 - 8 } & $\begin{array}{c}\text { Time } \\
\text { (s) }\end{array}$ & $\begin{array}{c}\text { Extracted } \\
\text { point } \\
\text { numbers }\end{array}$ & $\begin{array}{c}\text { Traversed } \\
\text { point } \\
\text { numbers }\end{array}$ & $\begin{array}{c}\text { Time } \\
\text { (s) }\end{array}$ & $\begin{array}{c}\text { Break } \\
\text { point } \\
\text { numbers }\end{array}$ & $\begin{array}{c}\text { Time } \\
\text { (s) }\end{array}$ & $\begin{array}{c}\text { Traversed } \\
\text { point } \\
\text { numbers }\end{array}$ \\
\hline FIG.7(A1) & 0.39 & 2397 & 15300 & 1.22 & 23 & 0.27 & 15043 \\
\hline FIG.7(B1) & 0.29 & 1783 & 16909 & 0.75 & 16 & 0.28 & 14717 \\
\hline FIG.7(C1) & 0.38 & 2694 & 21083 & 1.47 & 29 & 0.31 & 16262 \\
\hline FIG.7(D1) & 0.34 & 3103 & 18920 & 1.23 & 26 & 0.35 & 17772 \\
\hline FIG.8(A1) & 0.68 & 6550 & 28119 & 3.88 & 96 & 1.06 & 35837 \\
\hline FIG.8(B1) & 0.72 & 6380 & 28856 & 4.45 & 101 & 1.25 & 38796 \\
\hline FIG.8(C1) & 0.92 & 6403 & 35491 & 4.44 & 107 & 1.27 & 39133 \\
\hline FIG.8(D1) & 0.49 & 5509 & 22935 & 2.34 & 60 & 0.78 & 30089 \\
\hline
\end{tabular}

As no parallization technique is applied to accelerate the calculation of the convexity and symmetric metrics, 3-D image datasets need much more computation (between 10 and 100 seconds for 3D volumes with 300 to 700 slices sized $512 \times 512$ pixels). From Table 8, we found that the number of 
Fig. 11. Results on four synthetic 3D data volumes. Images in the first row are the input data volumes. Images in the second row represents the centerline extraction result while images in the third row shows the segmentation results.

Fig. 12 Results when varying $N$. (a) the retinal vessel image chosen from the DRIVE data base. (b) extraction result without using the local operation. (c), (d) and (e) results when $N$ equals 1000, 2000, and 5000, respectively. $\left(\alpha=0.7, l_{\mathrm{AVE}}=1000, \kappa=1, \lambda=20, l_{\mathrm{EV}}=10000, l_{\mathrm{bk}}=10, l_{\mathrm{EC}}=2500, N \bar{S}_{0_{-} \mathrm{c}}^{\min }=0.05, N \bar{S}_{0_{-}}^{\min }=0.05\right.$

$, R_{\min }=2, R_{\max }=10$ )

traversed points and the radius range are closely related to the computation complexity.

\subsection{Parameter analysis}

Though there are 12 parameters to be set in the proposed method for extracting the centerlines and vessel structures, their
$R_{\text {min }}$ and $R_{\text {max }}$ provide a rough range of target vessel radius. Their settings are also examined.

Fig.12 illustrates the extracted centerlines when different $N$ values are used in the local MPP-BT operation.We can see in the second column in Fig.12 (b) that the original MPP-BT
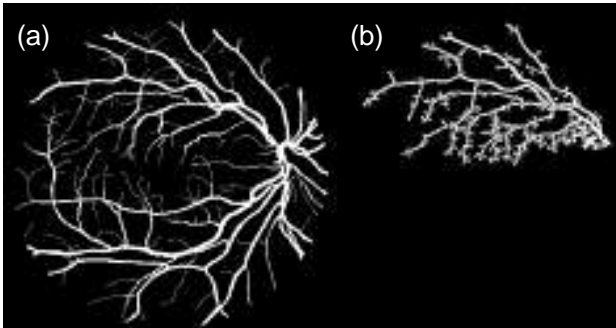

choice is not difficult. For all experimental datasets, six parameters are set to the same values (see Table 1). The other 6 parameters need an adjustment for different datasets but they remain unchanged for the same dataset type. The parameters $l_{\mathrm{bk}}$ $, l_{\mathrm{EC}}, N \bar{S}_{0_{-} \mathrm{c}}^{\mathrm{min}}$ for centerline extraction have been discussed in our previous work [30] so, in this section, the parameter $N$ for the local combination and the parameter $l_{\mathrm{EV}}$ for the stopping
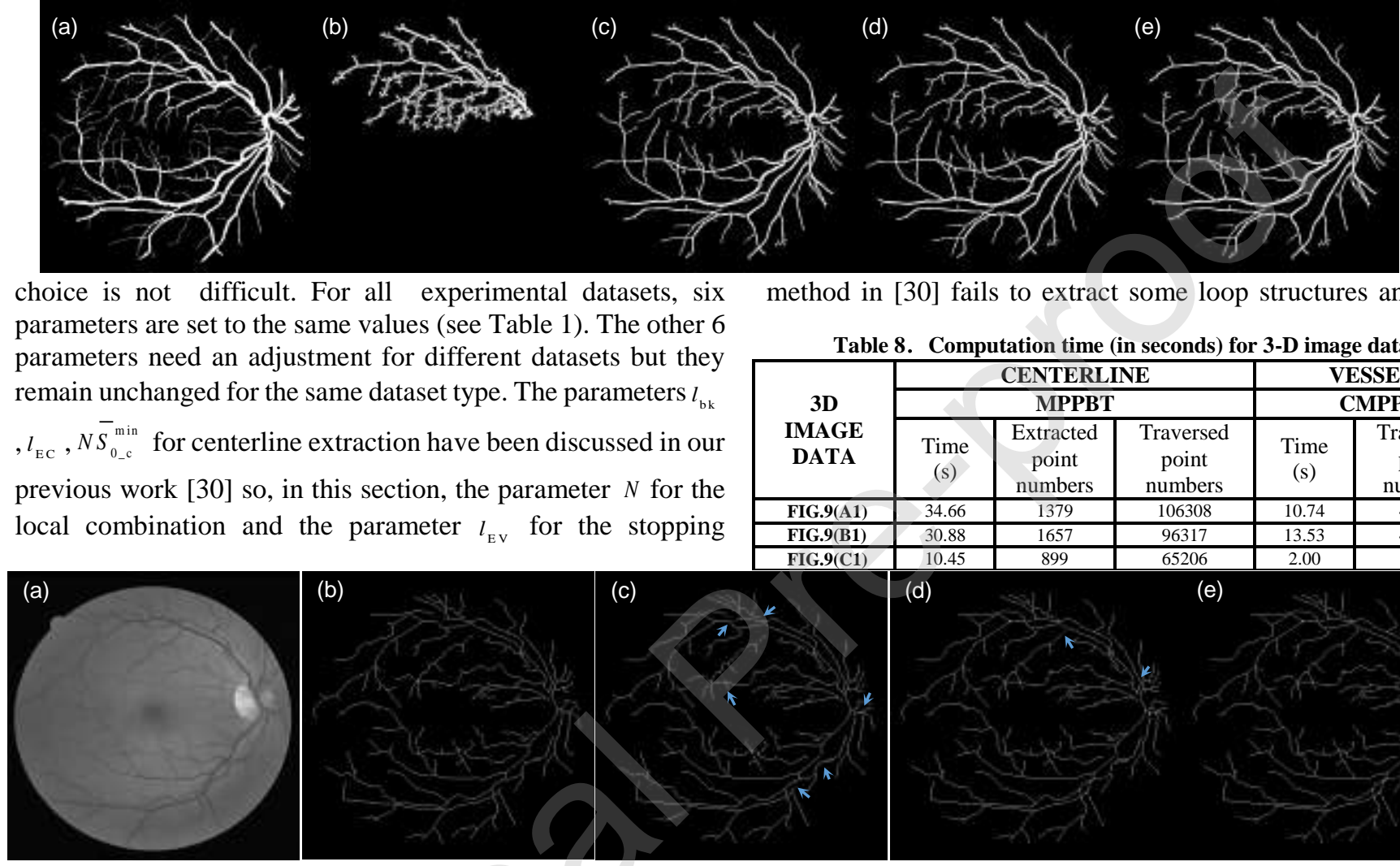

method in [30] fails to extract some loop structures and new

Table 8. Computation time (in seconds) for 3-D image data

\begin{tabular}{|c|c|c|c|c|c|}
\hline \multirow{2}{*}{$\begin{array}{c}\text { 3D } \\
\text { IMAGE } \\
\text { DATA }\end{array}$} & \multicolumn{3}{|c|}{ CENTERLINE } & \multicolumn{2}{c|}{ VESSEL } \\
\cline { 2 - 6 } & $\begin{array}{c}\text { Time } \\
(\mathrm{s})\end{array}$ & $\begin{array}{c}\text { Extracted } \\
\text { point } \\
\text { numbers }\end{array}$ & $\begin{array}{c}\text { Traversed } \\
\text { point } \\
\text { numbers }\end{array}$ & $\begin{array}{c}\text { Time } \\
(\mathrm{s})\end{array}$ & $\begin{array}{c}\text { Traversed } \\
\text { point } \\
\text { numbers }\end{array}$ \\
\hline FIG.9(A1) & 34.66 & 1379 & 106308 & 10.74 & 43786 \\
\hline FIG.9(B1) & 30.88 & 1657 & 96317 & 13.53 & 44071 \\
\hline FIG.9(C1) & 10.45 & 899 & 65206 & 2.00 & 8934 \\
\hline
\end{tabular}

criterion of vessel extraction are examined below. Parameter
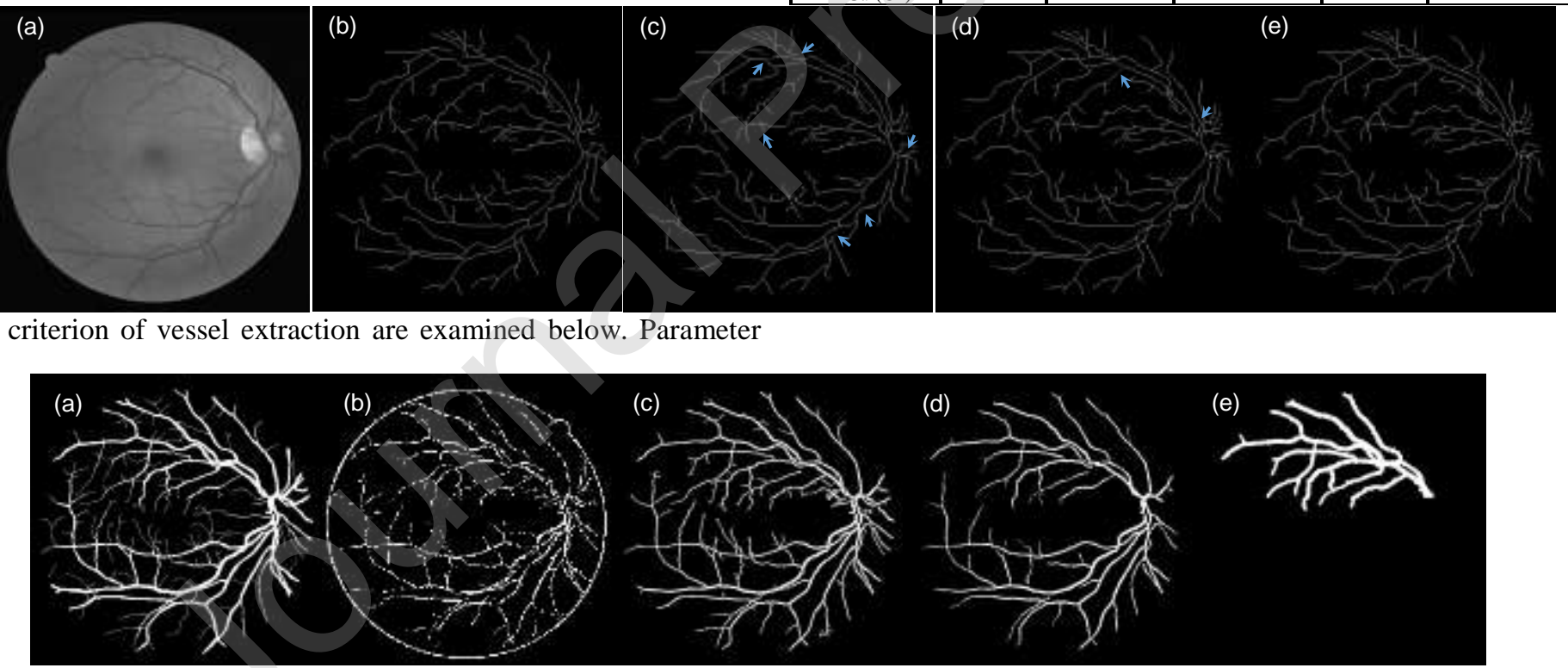

Fig. 13. Results on a retinal image with different $R_{\min }$. (a) Ground truth. (b) $R_{\min }=1$. (c) $R_{\min }=2$. (d) $R_{\min }=4$. (e) $R_{\min }=6$. We set $R_{\max }=10$ in all experiments here and other parameters are set according to Table 1.

Fig. 14. Results on a retinal image with different $R_{\max }$. (a) Ground truth. (b) $R_{\max }=6$. (c) $R_{\max }=8$. (d) $R_{\max }=10$. (e) $R_{\max }=15$. We set $R_{\min }=2$ in all experiments here and other parameters are set according to Table 1

structure increments (in red in Fig.12 (c)-(e)) can be brought by means of the additional local MPP-BT operations. Fig.12 (c)(e) shows that the local MPP-BT operation works well in extracting more close loop structures (with $N$ equals to 1000 , 

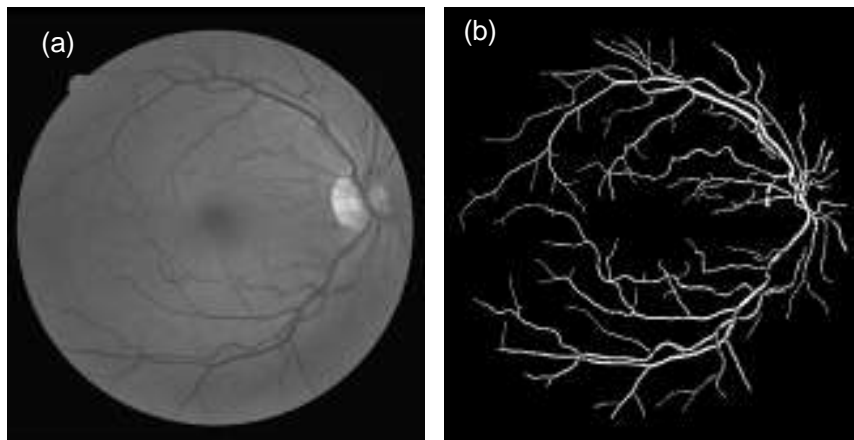

6 closed loops marked by the blue arrows in Fig.12(c) were extracted). When $N$ is increased to 2000, another 2 closed loops were found (also identified by the blue arrows in Fig.12 (d)). However, a larger value of $N$ does not bring any new benefit (see Fig.12 (e), and Table 9). In our experiments, $N$ was set to 2000 to achieve a good balance between the extraction accuracy and the computation cost.

Table 9. Relationship between $N$ and the computation cost

\begin{tabular}{|c|c|c|c|c|c|}
\hline Value of $\boldsymbol{N}$ & 1000 & 2000 & 3000 & 4000 & 5000 \\
\hline $\begin{array}{c}\text { Computation } \\
\text { Time (second) }\end{array}$ & 2.37 & 3.79 & 5.46 & 7.16 & 8.82 \\
\hline
\end{tabular}

Fig.16 illustrates the results obtained when varying $l_{\mathrm{EV}}\left(l_{\mathrm{EV}}\right.$ is used to modulate the minimal path propagation of vessel extraction). Because all the grid points traversed in the propagation belong to the final result points in some specific backtracking path, the choice of $l_{\mathrm{EV}}$ value has a large impact on the final extraction. Lowering the value of $l_{\mathrm{EV}}$ leads to miss edge points while increasing the value of $l_{\mathrm{EV}}$ tends to augment the false non-vessel points (marked by blue arrow) in the final result. Fig. 15 and Table 10 show the relationship between the values of $l_{\mathrm{EV}}$ and the values of Acc, Sp and Se. As the $l_{\mathrm{EV}}$ turns larger, the $\mathrm{Sp}$ value decreases and Se value increases. Acc reaches its largest value at around 10000 points are searched.

The extracted vascular structures with different $R_{\min }$ and $R_{\max }$ values in the local MPP-BT method are illustrated in Fig.13 and Fig.14. We vary $R_{\max }$ or $R_{\min }$ while the other parameters were kept unchanged (refer to Table 1). We can observe an oversegmentation of retinal edge with low $R_{\min }$ value in Fig.13(b) and a partial segmentation of the vessel structure with high
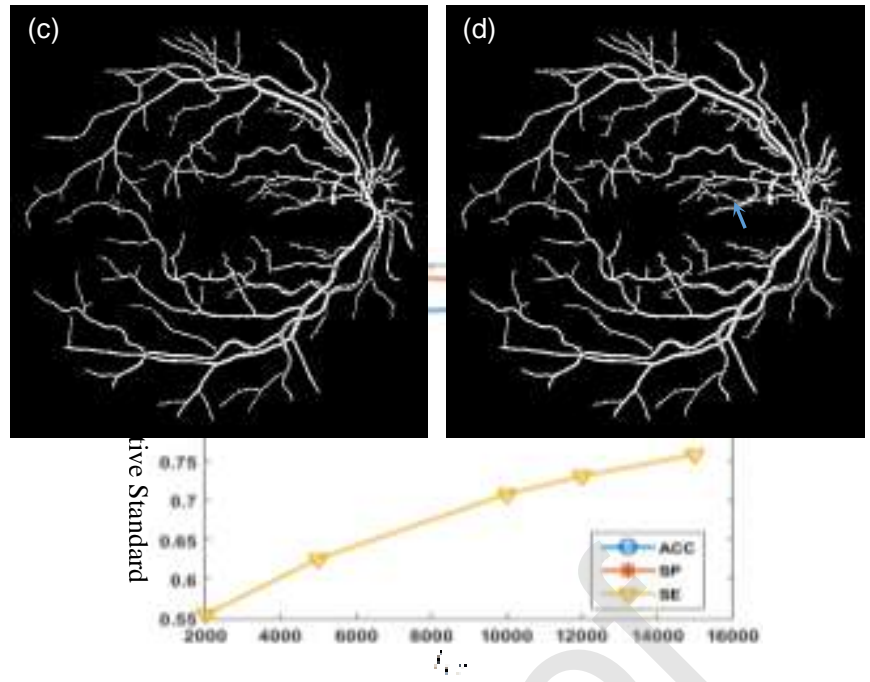

$R_{\min }$ in Fig.13(e). Fig.14(b) shows the incomplete vessel structure result extracted by local MPP-BT operation when the $R_{\max }$ value is high. Table 11 and Table 12 present the evolutions of Acc, Sp and Se when varying $R_{\min }, R_{\max }$. Large or small $R_{\min }$ values or small $R_{\max }$ values can lead to low values of Acc, Sp and Se For large $R_{\max }$ values, the change in accuracy is not significant. However, larger $R_{\max }$ values will inevitably involve higher computation cost.

Fig. 15 The relationship between the values of and quantitative standard ACC, SP, and SE of the result of the Fig.12 (a) using the proposed method.

Table 10. Quantitative evaluation when varying $l_{\mathrm{EV}}$

\begin{tabular}{|c|c|c|c|}
\hline$l_{E V}$ & Acc & Sp & Se \\
\hline $\mathbf{2 0 0 0}$ & 0.9348 & $\mathbf{0 . 9 9 2 2}$ & 0.5543 \\
\hline $\mathbf{5 0 0 0}$ & 0.9404 & 0.9879 & 0.6257 \\
\hline $\mathbf{1 0 0 0 0}$ & $\mathbf{0 . 9 4 4 2}$ & 0.9798 & 0.7082 \\
\hline $\mathbf{1 2 0 0 0}$ & 0.9440 & 0.9762 & 0.7309 \\
\hline $\mathbf{1 5 0 0 0}$ & 0.9426 & 0.9704 & $\mathbf{0 . 7 5 8 8}$ \\
\hline
\end{tabular}

Table 11. Quantitative evaluation when varying $R_{\min }$

\begin{tabular}{|c|c|c|c|}
\hline$R_{\min }$ & ACC & Se & Sp \\
\hline 1 & 0.9228 & 0.5321 & 0.9674 \\
\hline 2 & $\mathbf{0 . 9 5 5 7}$ & $\mathbf{0 . 6 7 2 2}$ & 0.9881 \\
\hline 4 & 0.9538 & 0.5832 & $\mathbf{0 . 9 9 6 1}$ \\
\hline 6 & 0.9008 & 0.2729 & 0.9725 \\
\hline
\end{tabular}

Fig. 16 Segmentation results with different $l_{E V}$ values on a retinal image. (a) The original retinal image. (b), (c) and (d) illustrate the extraction results when the $l_{E V}=2000, l_{E V}=10000$ and $l_{E V}=12000$, respectively. Other parameters are set according to Table 1 .

Fig. 17 Illustration on the effect of potential constraint and radius constraint. (a) the selected retinal vessel image; (b) the centerlines extracted by the proposed method; (c) result without using potential constraint and radius constraint; (d) result obtained with only the potential constraint (e) vessel extraction using both the

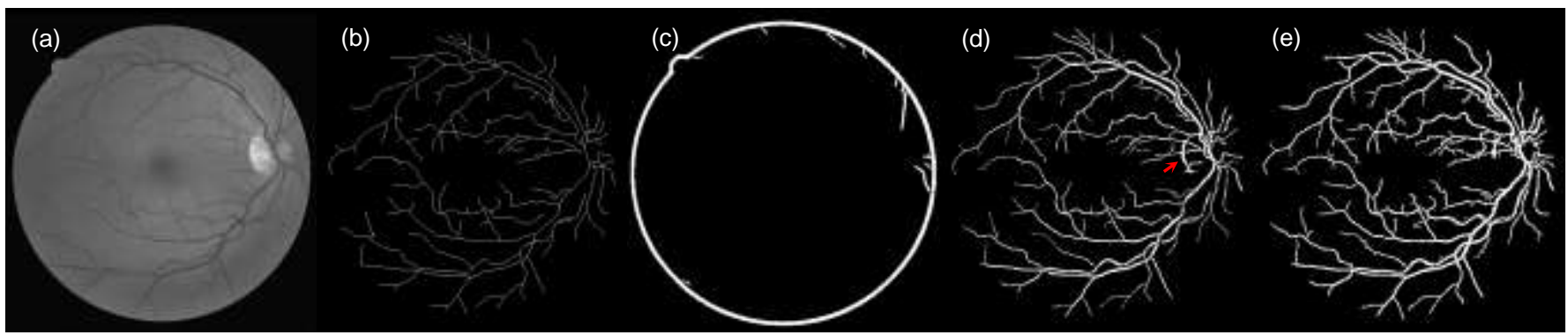


potential constraint and the radius constraint. $\left(\alpha=0.7, l_{\mathrm{AVE}}=1000, \kappa=1, \lambda=20, N=2000, l_{\mathrm{EV}}=10000, l_{\mathrm{bk}}=10, l_{\mathrm{EC}}=2500, N \bar{S}_{0 \mathrm{c}}^{\text {min }}=0.05, N \bar{S}_{0 \mathrm{v}}^{\text {min }}=0.05\right.$,

$$
R_{\min }=2, R_{\max }=10 \text { ). }
$$

Table 12. Quantitative evaluation when varying $R_{\max }$

\begin{tabular}{|c|c|c|c|}
\hline$R_{\max }$ & ACC & Se & Sp \\
\hline 6 & 0.9027 & 0.2619 & 0.9758 \\
\hline 8 & 0.9557 & 0.6725 & 0.9881 \\
\hline 10 & 0.9557 & 0.6722 & 0.9881 \\
\hline 15 & 0.9557 & 0.6723 & 0.9881 \\
\hline
\end{tabular}

\subsection{Analysis on potential constraint and radius constraint}

The effect of potential constraint and radius constraint in the proposed algorithm is also analyzed. Fig.17(a) to Fig.17(e) depict a retinal image selected from the DRIVE database, the centerline extraction result, the vessel extraction result without the constraints, the vessel extraction result with only the potential constraint and the vessel extraction result with the both constraints. From the Fig.17(c) we can see that without the constraints, the propagation falled into non-vessel regions with high ${ }^{M_{v}}$ values. Fig.17(d) shows that the potential constraint works well in limiting the vessel structures in the vessel regions. Also, the radius constraint allows constraining the propagation around the vessel regions. Thus, inclusion of non-vessel points is reduced (as observed by comparing the structures pointed by the red arrow in Fig.17(d) and Fig.17(e)).

\section{CONCLUSION AND FUTURE WORK}

In this paper, as an extension of our previous work on curvelike structure extraction using the MPP-BT method [30], we developed a method termed constraint-minimal path propagation (CMPP) to realize the extraction of complete vessel structures. Using the extracted centerline information as constraint, another minimal path propagation is performed for the vessel structure extraction. The proposed method is unsupervised and requires only one roughly set start point to automatically get the centerline and the vessel structures. The method is suitable for different kinds of vessel images including retinal images, coronary images and etc. In addition, by using local MPP-BT operations, the CMPP method greatly alleviate the structutre missing phenomenon for the close loop problems.

Different types of vessel data including the coronary artery, retinal vessel, kidney artery and the carotid artery are used in algorithm evaluatation. Visual illustrations in Fig.7 to Fig.9 show that the proposed method can provide an effective vessel extraction. Quantitative comparisons using retinal data also validate the effectiveness of this CMPP approach when compared to other supervised and unsupervised methods. It is also found that the involved 12 parameters can be set to the same values for the same type of data.

However, some issues should be addressed in the near future. Firstly, the local MPP-BT may not only extract the loop structures but also connects some branches in some 2D cases where branches are very close. Secondly, a smooth surface of the lumen is not ensured by the algorithm as there is no surface smoothness constraint in the proposed algorithm. Future work will look for solutions to this problem that do not penalize too much the computation cost. Thirdly, the lower convexity values of vessel boundary points leads to compromised segmentation specificity. A full study of pathological situations (such as calcifications, aneurysms or vessel stenosis) must also be performed. Such effort is not easy because we need ground truth data for assessment. Also, there are too many parameters to tune in the proposed algorithm. Although we provided some recommended parameter settings, an automatic parameter selection algorithm is needed in the future. Finally, regarding the 3D applications, the computation time, although not too high (about 40 seconds), needs to be reduced using acceleration techniques.

conflict of interests

The authors have declared that no conflict of interest exists.

\section{REFERENCES}

[1] McInerney T and Terzopoulos D. Deformable models in medical image analysis: A survey. Medical image analysis 1996; 1(2): 91-108. [2] Duncan, James S, and Ayache N. Medical image analysis: Progress over two decades and the challenges ahead. IEEE Transactions on Pattern Analysis and Machine Intelligence 2000; 22(1): 85-106.

[3] Pham, Dzung L, Xu CY, and Prince JL. Current methods in medical image segmentation 1. Annual review of biomedical engineering 2000; 2(1): 315-337.

[4] Kirbas, Cemil and Quek F. A review of vessel extraction techniques and algorithms. ACM Computing Surveys (CSUR) 2004; 36(2): 81-121.

[5] Lesage, David, et al. A review of 3D vessel lumen segmentation techniques: Models, features and extraction schemes. Medical image analysis 2009;13(6): 819-845.

[6] Frangi AF, Niessen WJ, Vincken KL, et al. Multiscale vessel enhancement filtering. Berlin Heidelberg: Springer 1998: 130-137.

[7] Lorigo LM, et al. CURVES: curve evolution for vessel segmentation. Medical Image Analysis 2001; 5(3): 195-206.

[8] Nain D, Yezzi A, and Turk G. Vessel Segmentation Using a Shape Driven Flow. Lecture Notes in Computer Science 2004; 3216: 51-59.

[9] Li C, et al. Minimization of region-scalable fitting energy for image segmentation. IEEE Transactions on Image Processing A Publication of the IEEE Signal Processing Society 2008; 17(10):19401949.

[10] Brieva J, Gonzalez E, Gonzalez F, et al. A level set method for vessel segmentation in coronary angiography. Conference: International Conference of the IEEE Engineering in Medicine \& Biology Society IEEE Engineering in Medicine \& Biology Society Conference. PubMed 2005; 6348-6351.

[11] Sum K, Cheung P. Vessel extraction under non-uniform illumination: a level set approach. IEEE transactions on bio-medical engineering 2008; 55(1): 358-360.

[12] Fan Z, Lu J, Wei C et al. A hierarchical image matting model for blood vessel segmentation in fundus images. IEEE Transactions on Image Processing 2019; 28(5): 2367-2377;

[13] Staal J, et al. Ridge-based vessel segmentation in color images of the retina. IEEE Transactions on Medial Imaging 2004; 23(4): 501509.

[14] Marín D, et al. A new supervised method for blood vessel segmentation in retinal images by using gray-level and moment invariants-based features. IEEE Transactions on Medical Imaging 2011: 30(1): 146-158. 
[15] Sayed M, Saha S, et al.: A Semi-supervised Approach to Segment Retinal Blood Vessels in Color Fundus Photographs. Conference on Artificial Intelligence in Medicine in Europe. Springer, Cham, 2019: 347-351.

[16] Alom, Hasan, Yakopcic, et al. Recurrent Residual Convolutional Neural Network based on U-Net (R2U-Net) for Medical Image Segmentation. arXiv:1802.06955.

[17] Zhuang J. LadderNet: Multi-path networks based on U-Net for medical image segmentation. arXiv:1810.07810.

[18] Fu H, Lin S, et al. DeepVessel: Retinal Vessel Segmentation via Deep Learning and Conditional Random Field. In MICCAI, 2016: 132139.

[19] Schaap, Michiel, et al. Bayesian tracking of elongated structures in 3D images. Biennial International Conference on Information Processing in Medical Imaging. Springer Berlin Heidelberg, 2007: 7485

[20] Li H, and Yezzi A. Vessels as 4-D curves: Global minimal 4-D paths to extract 3-D tubular surfaces and centerlines. IEEE Transactions on Medical Imaging 2007;26(9): 1213-1223.

[21] Benmansour, Fethallah, and Cohen LD. Tubular structure segmentation based on minimal path method and anisotropic enhancement. International Journal of Computer Vision 2011;92(2): 192-210.

[22] Wink, Onno, Niessen WJ, and Viergever WA. Multiscale vessel tracking. IEEE Transactions on Medical Imaging 2004;23(1): 130-133. [23] Aylward, Stephen R, and Bullitt E. Initialization, noise, singularities, and scale in height ridge traversal for tubular object centerline extraction. IEEE transactions on medical imaging 2002;21(2): 61-75.

[24] Gülsün, Akif M, and Tek H. Robust vessel tree modeling. International Conference on Medical Image Computing and Computer-Assisted Intervention. Springer Berlin Heidelberg, 2008: 602-611.

[25] Metz CT, et al. Coronary centerline extraction from CT coronary angiography images using a minimum cost path approach. Medical physics 2009; 36(12): 5568-5579.

[26] Aylward SR and Bullitt E. Initialization, noise, singularities, and scale in height ridge traversal for tubular object centerline extraction. IEEE transactions on medical imaging 2002; 21(2): 61-75.

[27] Li H, Yezzi A, and Cohen LD. 3D multi-branch tubular surface and centerline extraction with 4D iterative key points. International Conference on Medical Image Computing and Computer-Assisted Intervention. Springer Berlin Heidelberg, 2009: 1042-1050.

[28] Cohen LD, and Deschamps T. Segmentation of 3D tubular objects with adaptive front propagation and minimal tree extraction for 3D medical imaging. Computer methods in biomechanics and biomedical engineering. 2007: 10(4): 289-305.

[29] Kaul V, Yezzi A, and Tsai YC. Detecting curves with unknown endpoints and arbitrary topology using minimal paths. IEEE transactions on pattern analysis and machine intelligence. 2012; 34(10): 1952-1965.

[30] Chen Y, et al. Curve-like structure extraction using minimal path propagation with backtracking. IEEE Transactions on Image Processing 2016; 25(2): 988-1003.

[31] Chen Y, et al. Centerline constrained minimal path propagation for vessel extraction. 2014 IEEE 11th International Symposium on Biomedical Imaging (ISBI). IEEE, (Academic, 2014).

[32] Da C, Zhang J , and Cohen LD. Minimal paths for tubular structure segmentation with coherence penalty and adaptive anisotropy. IEEE Transactions on Image Processing 2018. 28(4): 1271-1284.

[33] Dijkstra, Edsger W. A note on two problems in connexion with graphs. Numerische mathematic 1959; 1(1): 269-271.

[34] Garcia M-P., et al. Coronary vein tracking from MSCT using a minimum cost path approach. 2010 IEEE International Symposium on Biomedical Imaging: From Nano to Macro. IEEE, (Academic, 2010).
[35] Niemeijer $M$, et al. Comparative study of retinal vessel segmentation methods on a new publicly available database. Medical Imaging 2004. International Society for Optics and Photonics, (Academic , 2004).

[36] Hoover A D, Kouznetsova V, Goldbaum M. Locating blood vessels in retinal images by piecewise threshold probing of a matched filter response[J]. IEEE Transactions on Medical imaging, 2000, 19(3): 203-210.

[37] Yang GY, et al. Characterization of 3-D coronary tree motion from MSCT angiography. IEEE Transactions on Information Technology in Biomedicine 2010; 14(1): 101-106.

[38] Hameeteman K, et al. Evaluation framework for carotid bifurcation lumen segmentation and stenosis grading. Medical Image Analysis 2011; 15(4): 477-488.

[39] NVIDIA CUDATM Programming Guide (Version 3.0), http://developer.download.nvidia.com/compute/cuda/3.0/toolkit/docs/ NVIDIA CUDA Programming Guide.pdf.

[40] Accelerating MATLAB with CUDA Using MEX Files (White Paper), http://developer.nvidia.com/object/matlab cuda.html.

[41] Chaudhuri S, et al. Detection of blood vessels in retinal images using two-dimensional matched filters. IEEE Transactions on Medical Imaging 1989; 8(3): 263-269.

[42] Zhang B, et al. Retinal vessel extraction by matched filter with first-order derivative of Gaussian. Computers in biology and medicine 2010; 40(4): 438-445.

[43] Espona L, et al. Retinal vessel tree segmentation using a deformable contour model. Pattern Recognition, 2008. ICPR 2008. 19th International Conference on. IEEE, (Academic, 2008).

[44] Mendonca, Maria A, and Campilho A. Segmentation of retinal blood vessels by combining the detection of centerlines and morphological reconstruction. IEEE Transactions on Medical Imaging 2006; 25(9): 1200-1213.

[45] Vlachos M, and Dermatas E. Multi-scale retinal vessel segmentation using line tracking. Computerized Medical Imaging and Graphics 2010; 34(3): 213-227.

[46] Hamarneh G, Jassi P. VascuSynth: simulating vascular trees for generating volumetric image data with ground-truth segmentation and tree analysis. Comput Med imaging Graph 2010; 34:605-616.

Guanyu Yang received the B.S. and M.S. degrees in biomedical engineering from Southeast University, Nanjing, China, in 2002 and 2005 respectively, and the Ph.D. degree in signal and image processing from the University of Rennes, Rennes, France, in 2009. He is currently an Associate Professor with the School of Computer Science and Engineering, Southeast University, Nanjing, China. His research interests are mainly focused on medical image processing and computerassisted systems for diagnosis and therapy in medicine.

Tianling Lv received the B.S. degree from Southeast University in 2015 . He is currently pursuing the Ph.D. degree in computer science with Southeast University. His interests are in image processing and medical image analysis. He is now working on automatic vessel structure segmentation algorithms.

Yunpeng Shen received the B.S. degree in biomedical engineering with Southeast University, in 2017. His interests are in image processing and medical image analysis. He is mainly working on vessel structure extraction algorithms.

Shuo Li is currently the Founding Scientific Director with Digital Imaging Group of London. His research interests 
include big data, cloud computing, machine learning, medical image analysis and computer aided diagnosis.

Jian Yang received the Ph.D. degree in optical engineering from the Beijing Institute of Technology, in 2007. He was a Post-Doctoral Research Fellow with the Mouse Imaging Centre, Hospital for Sick Children, Toronto, Canada, from 2007 to 2009. He is currently a Professor with the School of Optoelectronics, Beijing Institute of Technology, China. His research interests include medical image processing, computer vision, virtual reality, and augmented reality.

Yang Chen received the M.S. and Ph.D. degrees in biomedical engineering from First Military Medical University, China, in 2004 and 2007, respectively. Since 2008, he has been a Faculty Member with the Department of Computer Science and Engineering, Southeast University, China. His recent work concentrates on the medical image reconstruction, image analysis, pattern recognition, and computerized-aid diagnosis.

Huazhong Shu received the B.S. degree in applied mathematics from Wuhan University, China, in 1987, and the $\mathrm{Ph} . \mathrm{D}$. degree in numerical analysis from the University of Rennes, France, in 1992. He is currently a Professor with the Department of Computer Science and Engineering, Southeast University, China. His recent work concentrates on the image analysis, pattern recognition, and fast algorithms of digital signal processing.

Limin Luo received the Ph.D. degree from the University of Rennes, Rennes, France, in 1986. He is currently a Professor with the Department of Computer Science and Engineering, Southeast University, Nanjing, China. His current research interests include medical imaging, image analysis, computerassisted systems for diagnosis and therapy in medicine, and computer vision.

Jean-Louis Coatrieux received the Ph.D. and State Doctorate degrees in sciences from the University of Rennes 1, Rennes, France, in 1973 and 1983, respectively. He is now a professor of the Department of Computer Science and Engineering of Southeast University, China. His recent work concentrates on image analysis, pattern recognition, and fast algorithms of digital signal processing. 